Research Article

\title{
Picture Fuzzy TOPSIS Method Based on CPFRS Model: An Application to Risk Management Problems
}

\author{
Jiulin Jin (D, Ping Zhao, and Taijie You \\ School of Mathematical Sciences, Guizhou Normal University, Guiyang 550001, Guizhou, China \\ Correspondence should be addressed to Jiulin Jin; jliin@gznu.edu.cn
}

Received 10 December 2020; Revised 1 February 2021; Accepted 1 March 2021; Published 18 March 2021

Academic Editor: Jiwei Huang

Copyright ( $\odot 2021$ Jiulin Jin et al. This is an open access article distributed under the Creative Commons Attribution License, which permits unrestricted use, distribution, and reproduction in any medium, provided the original work is properly cited.

\begin{abstract}
It is not widely acknowledged that multicriteria decision-making (MCDM) problems in some particular circumstances cannot be effectively solved by some traditional methods. This paper aims to construct a novel decision-making method to effectively solve these MCDM problems. Firstly, we propose a covering-based picture fuzzy rough set (CPFRS) model by combining the picture fuzzy (PF) neighborhood operator and some PF logical operators. Secondly, by combining the proposed CPFRS model with the principle of TOPSIS method, a new method is proposed to solve the MCDM problems under PF environments. Finally, we apply our proposed method to the risk management of green buildings. By comparing the proposed method with some existing MCDM methods, the established method is effective and flexible and can be applied to a wide range of environments.
\end{abstract}

\section{Introduction}

Data-driven methods and techniques are significant tools and have been widely used in decision making [1]. Among many data-driven methods, methods related to multicriteria decision making (MCDM) play a key role in knowledge processing, information management, data mining, etc. However, with the rapid development of the information age and computer technology, massive and complex fuzzy data appear in various fields of human life. This gave rise to great challenges. For example, in the field of risk management of construction projects $[2,3]$, identifying the priority of risk factors through risk ranking is of great significance for improving the efficiency of risk management. Since the risk assessment information about construction projects is usually fuzzy and uncertain, the risk ranking is difficult to be implemented.

To describe fuzzy and uncertain information, the American cybernetic expert Zadeh [4] first proposed the notion of fuzzy set (FS) by using membership degree in 1965 . Compared with the classical set theory, FS theory has broken through the constraints of a two-valued logical system and has been widely used in modern science. However, with the in-depth study of the fuzzy set theory, it is found that FS based on a membership degree parameter is difficult to accurately describe the uncertainty of things. In 1986, Atanassov [5] proposed intuitionistic fuzzy set (IFS) based on three parameters of membership, nonmembership, and hesitation to describe fuzzy information. Some models in the intuitionistic fuzzy environment have been studied by a large number of scholars, and some remarkable results have been achieved [6-9]. At the same time, as the IFS theory gradually matures, its limitations gradually appear. Therefore, many scholars are committed to the expansion and development of IFS theory, and various expansion forms and their applications have been proposed, such as pythagorean fuzzy set theory [10-16], intuitionistic cubic fuzzy set theory [17], neutrosophic set theory [18-22], and so on.

Each neutrosophic set (NS) [18] has three membership functions: true membership function, uncertain membership function, and false membership function. Intuitively, NS has a wide range of applications. However, since the function values of the three functions of the NS are all subsets of the nonstandard unit interval $] 0^{-}, 1^{+}[$, it is difficult to solve practical problems. Thus, two important subclasses of NS, picture fuzzy set (PFS) [23] and spherical fuzzy set (SFS) [24], are proposed one after another, and they are both characterized by the degrees of positive 
membership, neutral membership, negative membership, and refusal membership. Recently, SFS and its applications [25-35] have been developed rapidly. The main difference between PFSs and SFSs is that, in the former case, the sum of positive membership degree, neutral membership degree, and negative membership degree belongs to the standard unit interval $[0,1]$. Hence, PFS is regarded as the standard NS [36].

PFS is a good advanced fuzzy set for modeling some phenomena and events that cannot be processed in other sets such as FS and IFS [37]. To describe fuzzy and uncertain information more accurately, PFS is applied to the information evaluation of MCDM. Methods for MCDM with fuzzy or intuitionistic fuzzy information are no longer suitable for solving the MCDM problem with picture fuzzy (PF) information. How to deal with the problem of MCDM under PF environments has attracted the attention of a large number of researchers $[3,38-44]$. There are three major families of methods of MCDM under PF environments. (1) The approaches based on measure theory. There are three main measure methods, which are entropy measure, similarity measure, and distance measure. For example, Wei [39] proposed PF cross-entropy and applied it to the selection of enterprise resource planning system. Joshi and Kumar [40] proposed an approach for MCDM problems based on the Dice similarity measure and weighted Dice similarity measure for PFS. Peng and Dai [41] proposed an algorithm to solve the MCDM problem based on a distance measure. Wang et al. [42] studied the Bonferroni average distance of PFS and applied it to the evaluation of energy performance contracting projects. (2) The approaches based on the classical decision-making methods. For example, Wang et al. [3] expanded the VIKOR method to picture fuzzy normalized projection model and established a MCDM framework for risk evaluation of construction projects with PF information. Wei [43] expanded the TODIM method to the MCDM with PF information. Ashraf et al. [44] expanded the TOPSIS method to the MCDM with PF information. (3) The approaches based on utility theory. The information fusion technique based on some aggregation operators is a classical method in this family; many researchers [44-56] have proposed aggregation operators to fuse information in decision making. Some common aggregation operators are shown as follows:

(i) Picture fuzzy weighted average operator [47-50]

(ii) Picture fuzzy weighted geometric operator $[44,48]$

(iii) Picture fuzzy Muirhead mean operator [51]

(iv) Weighted picture fuzzy power Choquet ordered geometric operator and weighted picture fuzzy power Shapley Choquet ordered geometric operator [52]

(v) Picture fuzzy weighted interaction aggregation operator [53]

(vi) Picture fuzzy weighted interaction geometric operator [54]

(vii) Picture fuzzy Dombi aggregation operator [55] (viii) Picture fuzzy Hamacher aggregation operator $[47,56]$

Although some of the existing aggregation operators have been successfully applied to the MCDM problem under PF environments, they all have a common shortcoming, that is, they may not be able to deal with the MCDM problem in a finite picture fuzzy covering approximation space (PFCAS).

Rough set (RS) theory, as an effective tool for analyzing information with inaccuracy, inconsistency, and incompleteness, was first introduced by Pawlak [57]. The most significant difference between RS theory and other theories dealing with uncertain and imprecise problems is that it does not need to provide any prior information beyond the dataset to be processed by the problem, so the description or treatment of the uncertainty of the problem can be said to be more objective. However, the binary relation in the classical RS model is an equivalence, which is very demanding and limits the application of RS models. Therefore, RS theory has been extended in many ways [58-67], among which the technology of combining FS theory, covering theory, and RS theory is an important research topic, for example, coveringbased fuzzy rough set model [61-64], covering-based intuitionistic fuzzy rough set model $[65,66]$, covering-based spherical fuzzy rough set model [67], and so on.

Compared with FS and IFS, PFS with four dimensions of yes, abstention, no, and rejection has more advantages in describing fuzzy and uncertain information. This paper has the following two basic motivations:

(1) RS theory has advantages in handling uncertain information. However, the classical RS model is limited in applications due to its strict conditions. Through our research, we find that the introduction of PFS theory makes many problems involving positive degree, neutral degree, negative degree, and refusal degree. There are few studies on the intersection of PFS theory and RS theory. Therefore, we will try to propose a covering-based picture fuzzy rough set (CPFRS) model by combining PF theory, covering theory, and RS theory.

(2) In the construction industry, the project management team uses risk assessment to determine the priority of risk management, which can greatly improve the efficiency of risk management. This type of problem can be described as the MCDM problem in a finite PFCAS. Nonetheless, some methods may fail in a finite PFCAS, which can be seen from Example 2 of this paper. TOPSIS is one of the classical decision-making methods, which can make full use of the information of the original data and can accurately reflect the gap between the evaluation plans. Since the TOPSIS method is suitable for the calculation of small samples and the calculation of large samples, it has been successfully applied to MCDM problems in various complicated fuzzy environments $[13,35,44,65-69]$. We can see that the advantage of the CPFRS model and the TOPSIS method cannot be ignored. Therefore, we will try to 
combine them in dealing with uncertain information and design a new decision-making method to solve the risk management problem.

The main objective of this paper is to design a decisionmaking method to solve MCDM problems in a finite PFCAS. This paper has the following contributions:

(1) The neighborhood operator is introduced based on PF covering, and its properties are discussed.

(2) We propose a CPFRS model by using the neighborhood operator and some PF logical operators. The CPFRS model not only enriches the concept of RS theory but also expands the scope of applications of RS theory.

(3) We design an algorithm to tackle MCDM problems in a finite PFCAS and apply our proposed method to risk management of green building projects.

(4) We compare our proposed method with existing methods to show the effectiveness of the proposed method.

The remainder of this paper is organized as follows. In Section 2, we review some important concepts about PFS. In Section 3, we analyze the shortcomings of some MCDM methods and construct a CPFRS model based on the PF neighborhood operator and some PF logic operators. In Section 4, we establish an approach to MCDM problems with the evaluation of PF information based on the CPFRS model. In addition, we apply the proposed method to the risk management problem of green buildings and verify the effectiveness and scientificity of the method through comparative analysis. Finally, several conclusions and future research work are given in Section 5.

\section{Preliminary}

In this section, we recall some basic concepts, which are necessary background knowledge.

\subsection{Picture Fuzzy Set (PFS)}

Definition 1 (see [23]). Let $U$ be a nonempty universe; a PFS on $U$ is described as

$$
A=\left\{\left\langle x, \mu_{A}(x), \eta_{A}(x), v_{A}(x)\right\rangle: x \in U\right\},
$$

which is characterized by a positive membership function $\mu_{A}: U \longrightarrow[0,1]$, a neutral membership function $\eta_{A}: U \longrightarrow[0,1]$, and a negative membership function $v_{A}: U \longrightarrow[0,1]$ with the condition: for all $x \in U$,

$$
0 \leq \mu_{A}(x)+\eta_{A}(x)+v_{A}(x) \leq 1,
$$

and $\pi_{A}(x)=1-\mu_{A}(x)-\eta_{A}(x)-v_{A}(x)$ is called the refusal degree of $x$ in $A$. In addition, $\mathscr{F}(U)$ denotes the family of PFSs.

For convenience, we write the triplet $\alpha=\left\langle\mu_{\alpha}, \eta_{\alpha}, v_{\alpha}\right\rangle$ as a picture fuzzy number (PFN). Additionally, $\pi_{\alpha}=1-\mu_{\alpha}-\eta_{\alpha}-$ $v_{\alpha}$ is the refusal degree of the PFN $\alpha$.

Definition 2 (see [23]). Let $A, B \in \mathscr{F}(U)$. The basic operations between two PFSs are shown as follows:

(1) $A \subseteq B \Longleftrightarrow \mu_{A}(x) \leq \mu_{B}(x), \quad \eta_{A}(x) \leq \eta_{B}(x), \quad$ and $v_{A}(x) \geq v_{B}(x)$ for all $x \in U$

(2) $A=B \Longleftrightarrow A \subseteq B$ and $B \subseteq A$

(3) $A \cup B=\left\{\left\langle x, \max \quad\left\{\mu_{A}(x), \mu_{B}(x)\right\}, \min \quad\left\{\eta_{A}(x)\right.\right.\right.$, $\left.\left.\left.\eta_{B}(x)\right\}, \min \left\{v_{A}(x), v_{B}(x)\right\}\right\rangle: x \in U\right\}$

(4) $A \cap B=\left\{\left\langle x, \quad \min \left\{\mu_{A}(x), \mu_{B}(x)\right\}, \quad \min \left\{\eta_{A}(x), \eta_{B}\right.\right.\right.$ $\left.\left.(x)\}, \max \left\{v_{A}(x), v_{B}(x)\right\}\right\rangle: x \in U\right\}$

(5) $\bar{A}=\left\{\left\langle x, v_{A}(x), \eta_{A}(x), \mu_{A}(x)\right\rangle: x \in U\right\}$

Definition 3 (see [70]). Let $\mathbb{P}=\left\{\left\langle x_{1}, x_{2}, x_{3}\right\rangle \in[0,1]^{3}: x_{1}+\right.$ $\left.x_{2}+x_{3} \leq 1\right\}$ and $\left\langle x_{1}, x_{2}, x_{3}\right\rangle,\left\langle y_{1}, y_{2}, y_{3}\right\rangle \in \mathbb{P}$. The partial order $\leq_{\mathbb{P}}$ on $\mathbb{P}$ can be defined by

$$
\begin{aligned}
& \left\langle x_{1}, x_{2}, x_{3}\right\rangle \leq_{\mathbb{P}}\left\langle y_{1}, y_{2}, y_{3}\right\rangle \\
& \Longleftrightarrow\left(x_{1}<y_{1} \text { and } x_{3} \geq y_{3}\right) \text { or }\left(x_{1}=y_{1} \text { and } x_{3}>y_{3}\right) \\
& \text { or }\left(x_{1}=y_{1}, x_{3}=y_{3} \text { and } x_{2} \leq y_{2}\right) .
\end{aligned}
$$

Definition 4 (see [70]). Let $x=\left\langle x_{1}, x_{2}, x_{3}\right\rangle, y=\left\langle y_{1}, y_{2}\right.$, $\left.y_{3}\right\rangle \in \mathbb{P}$ and let

$$
\begin{aligned}
& \inf (x, y)= \begin{cases}x, & \text { if } x \leq_{\mathbb{P}} y, \\
y, & \text { if } y \leq_{\mathbb{P}} x, \\
\left\langle\min \left\{x_{1}, y_{1}\right\}, 1-\min \left\{x_{1}, y_{1}\right\}-\max \left\{x_{3}, y_{3}\right\}, \max \left\{x_{3}, y_{3}\right\}\right\rangle, & \text { otherwise, }\end{cases} \\
& \sup (x, y)= \begin{cases}y, & \text { if } x \leq_{\mathbb{P}} y, \\
x, & \text { if } y \leq_{\mathbb{P}} x, \\
\left\langle\max \left\{x_{1}, y_{1}\right\}, 0, \min \left\{x_{3}, y_{3}\right\}\right\rangle, & \text { otherwise. }\end{cases}
\end{aligned}
$$

Denote $1_{\mathbb{P}}=\langle 1,0,0\rangle, 0_{\mathbb{P}}=\langle 0,0,1\rangle$. Then, $\left(\mathbb{P}, \leq_{\mathbb{P}}\right)$ is a complete lattice.

Indeed, Cuong et al. [70] provided that for each nonempty set $A \subseteq \mathbb{P}, \inf A=\left\langle\theta_{1}, \theta_{2}, \theta_{3}\right\rangle$ and $\sup A=\left\langle\tau_{1}, \tau_{2}, \tau_{3}\right\rangle$, where $\quad \theta_{1}=\min \left\{x_{1}:\left(\exists x_{2}, x_{3} \in[0,1]\right)\left\langle x_{1}, x_{2}, x_{3}\right\rangle \in A\right\}$, $\theta_{3}=\max \left\{x_{3}:\left(\exists x_{1}, x_{2} \in[0,1]\right)\left\langle x_{1}, x_{2}, x_{3}\right\rangle \in A\right\}$, $\tau_{1}=\max \left\{x_{1}:\left(\exists x_{2}, x_{3} \in[0,1]\right)\left\langle x_{1}, x_{2}, x_{3}\right\rangle \in A\right\}$, $\tau_{3}=\min \left\{x_{3}:\left(\exists x_{1}, x_{2} \in[0,1]\right)\left\langle x_{1}, x_{2}, x_{3}\right\rangle \in A\right\}$, 
$\theta_{2}=\left\{\begin{array}{ll}1-\theta_{1}-\theta_{3} & \text { if }\left\langle\theta_{1}, z, \theta_{3}\right\rangle \notin A \text { for all } z \\ \min \left\{x_{2}:\left\langle\theta_{1}, x_{2}, \theta_{3}\right\rangle \in A\right\} & \text { otherwise }\end{array}\right.$ and $\tau_{2}= \begin{cases}0 & \text { if }\left\langle\tau_{1}, z, \tau_{3}\right\rangle \notin A \text { for all } z \\ \max \left\{x_{2}:\left\langle\tau_{1}, x_{2}, \tau_{3}\right\rangle \in A\right\} & \text { otherwise }\end{cases}$

\subsection{PF Logical Operators}

Definition 5 (see $[70,71,72]$ ). A PF negator is a decreasing mapping $\mathscr{N}: \mathbb{P} \longrightarrow \mathbb{P}$ which satisfies $\mathscr{N}\left(1_{\mathbb{P}}\right)=0_{\mathbb{P}}$ and $\mathcal{N}\left(0_{\mathbb{P}}\right)=1_{\mathbb{P}}$. In particular, when $\mathcal{N}(\mathcal{N}(\alpha))=\alpha$ for all $\alpha \in \mathbb{P}$, then $\mathcal{N}$ is called an involutive negator.

For each $x=\left\langle x_{1}, x_{2}, x_{3}\right\rangle \in \mathbb{P}$, we denote

$$
I(x)=\left\{\left\langle x_{1}, y, x_{3}\right\rangle \in \mathbb{P}: 0 \leq y \leq x_{2}\right\} .
$$

Definition 6 (see $[70,71,72]$ ). A PF $t$-norm $\mathscr{T}: \mathbb{P}^{2} \longrightarrow \mathbb{P}$ is an increasing, commutative, and associative mapping which satisfies $\mathscr{T}\left(1_{\mathbb{P}}, x\right) \in I(x)$ for all $x \in \mathbb{P}$. In particular, if a PF $t$-norm $\mathscr{T}$ satisfies $\mathscr{T}\left(1_{\mathbb{P}}, x\right)=x$ for each $x \in \mathbb{P}$, then $\mathscr{T}$ is called a PF strong $t$-norm.

Definition 7 (see $[70,71,72]$ ). A PF $t$-conorm $\delta: \mathbb{P}^{2} \longrightarrow \mathbb{P}$ is an increasing, commutative, and associative mapping which satisfies $\mathcal{S}\left(0_{\mathbb{P}}, x\right) \in I(x)$ for all $x \in \mathbb{P}$.

Definition 8 (see $[70,71,72]$ ). If a mapping $\mathscr{I}: \mathbb{P}^{2} \longrightarrow \mathbb{P}$ satisfies the following conditions:

(1) $\mathscr{I}\left(0_{\mathbb{P}}, 0_{\mathbb{P}}\right)=\mathscr{I}\left(0_{\mathbb{P}}, 1_{\mathbb{P}}\right)=\mathscr{I}\left(1_{\mathbb{P}}, 1_{\mathbb{P}}\right)=1_{\mathbb{P}}$ and $\mathscr{I}\left(1_{\mathbb{P}}, 0_{\mathbb{P}}\right)=0_{\mathbb{P}}$

(2) If $x \leq_{\mathbb{P}} y$, then $\mathscr{I}(x, z) \geq_{\mathbb{P}} \mathscr{I}(y, z)$ for all $x, y, z \in \mathbb{P}$

(3) If $x \leq_{\mathbb{P}} y$, then $\mathscr{I}(z, x) \leq_{\mathbb{P}} \mathscr{I}(z, y)$ for all $x, y, z \in \mathbb{P}$, then $\mathscr{I}$ is called a PF implicator. In particular,

(4) If $\mathscr{I}\left(1_{\mathbb{P}}, x\right) \leq_{\mathbb{P}} x$ for each $x \in \mathbb{P}$, then PF implicator $\mathscr{I}$ is called a border implicator.

(5) If $x \leq_{\mathbb{P}} y \Longleftrightarrow \mathscr{I}(x, y)=1_{\mathbb{P}}$ for each $x \in \mathbb{P}$, then $\mathscr{I}$ is said to satisfy the confinement law.

Definition 9 (see [72]). Let $\mathcal{S}$ be a PF $t$-conorm and $\mathcal{N}$ be a $\mathrm{PF}$ negator. For each $x, y \in \mathbb{P}$, a $\mathrm{PF} \mathcal{S}$-implicator $\mathscr{I}_{\mathcal{S}, \mathcal{N}}$ based on $\mathcal{S}$ and $\mathcal{N}$ is defined as

$$
\mathscr{I}_{\delta, \mathcal{N}}(x, y)=\mathcal{S}(\mathcal{N}(x), y)
$$

By Definition 8, it is simply to see that the following proposition is true.

Proposition 1. Let $\mathcal{S}$ be a PF t-conorm and $\mathcal{N}$ be a PF negator. Then, for each $x, y \in \mathbb{P}$,

$$
\mathscr{I}_{\mathcal{S}, \mathcal{N}}^{*}(x, y)= \begin{cases}1_{\mathbb{P}}, & \text { if } x \leq_{\mathbb{P}} y \\ \mathcal{S}(\mathscr{N}(x), y), & \text { otherwise }\end{cases}
$$

is a PF border implicator and it satisfies the confinement law.

Example 1. Some common PF logical operators [71, 72] are shown below: for all $x=\left\langle x_{1}, x_{2}, x_{3}\right\rangle, y=\left\langle y_{1}, y_{2}, y_{3}\right\rangle \in \mathbb{P}$,

(i) The standard PF negator:

$$
\mathcal{N}_{s}(x)=\left\langle x_{3}, 1-x_{1}-x_{2}-x_{3}, x_{1}\right\rangle \text {. }
$$

(ii) The infimum operator (PF strong $t$-norm):

$$
\mathscr{T}_{I}(x, y)=\inf (x, y) \text {. }
$$

(iii) The Łuksiewicz $t$-norm:

$$
\begin{aligned}
\mathscr{T}_{L}(x, y) & =\left\langle\max \left(0, x_{1}+y_{1}-1\right), \max \left(0, x_{2}+y_{2}-1\right),\right. \\
& \left.\min \left(1, x_{3}+y_{3}\right)\right\rangle .
\end{aligned}
$$

(iv) The standard maximum operator (PF $t$-conorm):

$$
\mathcal{S}_{M}(x, y)=\left\langle\max \left(x_{1}, y_{1}\right), \min \left(x_{2}, y_{2}\right), \min \left(x_{3}, y_{3}\right)\right\rangle .
$$

(v) The algebraic product operator (PF $t$-conorm):

$$
\mathcal{S}_{P}(x, y)=\left\langle x_{1}+y_{1}-x_{1} y_{1}, x_{2} y_{2}, x_{3} y_{3}\right\rangle
$$

(vi) The Łukasiewicz $t$-conorm:

$$
\begin{aligned}
\mathcal{S}_{L}(x, y) & =\left\langle\min \left(1, x_{1}+y_{1}\right), \max \left(0, x_{2}+y_{2}-1\right),\right. \\
& \left.\max \left(0, x_{3}+y_{3}-1\right)\right\rangle .
\end{aligned}
$$

\section{Covering-Based Picture Fuzzy Rough Set (CPFRS) Model}

The following first gives the relevant content in $[47-49,53,55,56]$ and analyzes their limitations in dealing with some complex MCDM problems.

Let $\alpha_{i}=\left\langle\mu_{i}, \eta_{i}, v_{i}\right\rangle(i=1,2, \ldots, n)$ be a number of PFNs that correlated with weight vector $\omega=\left(\omega_{1}, \omega_{2}, \ldots, \omega_{n}\right)$ such that $\sum_{i=1}^{n} \omega_{i}=1$ and $\omega_{i} \in[0,1]$ for all $i$. Some aggregation operators are shown as Definitions 10-14.

Definition 10 (see $[47,48]$ ). Three PF aggregation operators are listed as follows:

(1) $\operatorname{PFWA}\left(\alpha_{1}, \alpha_{2}, \ldots, \alpha_{n}\right)=\left\langle 1-\prod_{i=1}^{n}\left(1-\mu_{i}\right)^{\omega_{i}}\right.$, $\left.\prod_{i=1}^{n} \eta_{i}^{\omega_{i}}, \prod_{i=1}^{n} \nu_{i}^{\omega_{i}}\right\rangle$

(2) $\operatorname{PFOWA}\left(\alpha_{1}, \alpha_{2}, \ldots, \alpha_{n}\right)=\left\langle 1-\prod_{i=1}^{n}\left(1-\mu_{\sigma(i)}\right)^{\omega_{i}}\right.$, $\left.\prod_{i=1}^{n} \eta_{\sigma(i)}^{\omega_{i}}, \prod_{i=1}^{n} v_{\sigma(i)}^{\omega_{i}}\right\rangle$,

(3) $\operatorname{PFHWA}\left(\alpha_{1}, \alpha_{2}, \ldots, \alpha_{n}\right)=\left\langle 1-\prod_{i=1}^{n}\left(1-\tilde{\mu}_{\sigma(i)}\right)^{\omega_{i}}\right.$, $\left.\prod_{i=1}^{n} \widetilde{\eta}_{\sigma(i)}^{\omega_{i}}, \prod_{i=1}^{n} \widetilde{v}_{\sigma(i)}^{\omega_{i}}\right\rangle$. 
Definition 11 (see [49]). Three PF aggregation operators, which are provided by Wang, are listed as follows:

(1) $\operatorname{PFWA}^{*}\left(\alpha_{1}, \alpha_{2}, \ldots, \alpha_{n}\right)=\left\langle 1-\prod_{i=1}^{n}\left(1-\mu_{i}\right)^{\omega_{i}}\right.$, $\prod_{i=1}^{n}\left(\eta_{i}+\pi_{i}\right)^{\omega_{i}}-\prod_{i=1}^{n} \pi_{i}^{\omega_{i}}, \prod_{i=1}^{n}\left(1-\mu_{i}\right)^{\omega_{i}}-$ $\left.\prod_{i=1}^{n}\left(\eta_{i}+\pi_{i}\right)^{\omega_{i}}\right\rangle$

(2) $\operatorname{PFOWA}^{*}\left(\alpha_{1}, \alpha_{2}, \ldots, \alpha_{n}\right)=\left\langle 1-\prod_{i=1}^{n}\left(1-\mu_{\sigma(i)}\right)^{\omega_{i}}\right.$, $\prod_{i=1}^{n}\left(\eta_{\sigma(i)}+\pi_{\sigma(i)}\right)^{\omega_{i}}-\prod_{i=1}^{n} \pi_{\sigma(i)}^{\omega_{i}}, \prod_{i=1}^{n}\left(1-\mu_{\sigma(i)}\right)^{\omega_{i}}-$ $\left.\prod_{i=1}^{n}\left(\eta_{\sigma(i)}+\pi_{\sigma(i)}\right)^{\omega_{i}}\right\rangle$,

(3) $\operatorname{PFHWA}^{*}\left(\alpha_{1}, \alpha_{2}, \ldots, \alpha_{n}\right)=\left\langle 1-\prod_{i=1}^{n}\left(1-\tilde{\mu}_{\sigma(i)}\right)^{\omega_{i}}\right.$, $\prod_{i=1}^{n}\left(\widetilde{\eta}_{\sigma(i)}+\tilde{\pi}_{\sigma(i)}\right)^{\omega_{i}}-\prod_{i=1}^{n} \tilde{\pi}_{\sigma(i)}^{\omega_{i}}, \prod_{i=1}^{n}\left(1-\widetilde{\mu}_{\sigma(i)}\right)^{\omega_{i}}-$ $\left.\prod_{i=1}^{n}\left(\widetilde{\eta}_{\sigma(i)}+\tilde{\pi}_{\sigma(i)}\right)^{\omega_{i}}\right\rangle$.

Definition 12 (see $[47,56]$ ). Let $\gamma>0$. Three PF Hammer weighted averaging operators are listed as follows:

(1) $\operatorname{PFHWA}_{\gamma}\left(\alpha_{1}, \alpha_{2}, \ldots, \alpha_{n}\right)=\left\langle\left(\prod_{i=1}^{n}\left(1+(\gamma-1) \mu_{i}\right)^{\omega_{i}}-\right.\right.$ $\prod_{i=1}^{n}\left(1-\mu_{i}\right)^{\omega_{i}} / \prod_{i=1}^{n}\left(1+(\gamma-1) \mu_{i}\right)^{\omega_{i}}+(\gamma-1)$

$\left.\prod_{i=1}^{n}\left(1-\mu_{i}\right)^{\omega_{i}}\right),\left(\gamma \prod_{i=1}^{n} \eta_{i}^{\omega_{i}} / \quad \prod_{i=1}^{n}\right.$ $\left.\left(1+(\gamma-1)\left(1-\eta_{i}\right)\right)^{\omega_{i}}+(\gamma-1) \prod_{i=1}^{n} \eta_{i}^{\omega_{i}}\right),\left(\gamma \prod_{i=1}^{n} \nu_{\alpha_{i}}^{\omega_{i}} /\right.$ $\left.\left.\prod_{i=1}^{n}\left(1+(\gamma-1)\left(1-v_{\alpha_{i}}\right)\right)^{\omega_{i}}+(\gamma-1) \prod_{i=1}^{n} \nu_{\alpha_{i}}^{\omega_{i}}\right)\right\rangle$,

(2) $\operatorname{PFHOWA}_{\gamma}\left(\alpha_{1}, \alpha_{2}, \ldots, \alpha_{n}\right)=\left\langle\left(\prod_{i=1}^{n} \quad(1+(\gamma-1)\right.\right.$ $\left.\mu_{\sigma(i)}\right)^{\omega_{i}}-\prod_{i=1}^{n}\left(1-\mu_{\sigma(i)}\right)^{\omega_{i}} / \prod_{i=1}^{n}\left(1+(\gamma-1) \mu_{\sigma(i)}\right)^{\omega_{i}}+$ $\left.(\gamma-1) \prod_{i=1}^{n}\left(1-\mu_{\sigma} \quad(i)\right)^{\omega_{i}}\right),\left(\gamma \prod_{i=1}^{n} \eta_{\sigma(i)}^{\omega_{i}} / \prod_{i=1}^{n}(1+\right.$ $\left.\left.(\gamma-1)\left(1-\eta_{\sigma(i)}\right)\right)^{\omega_{i}}+(\gamma-1) \prod_{i=1}^{n} \eta_{\sigma(i)}^{\omega_{i}}\right),\left(\gamma \prod_{i=1}^{n} \nu_{\sigma(i)}^{\omega_{i}}\right.$ $\left.\left./ \prod_{i=1}^{n}\left(1+(\gamma-1)\left(1-v_{\sigma(i)}\right)\right)^{\omega_{i}}+(\gamma-1) \prod_{i=1}^{n} \nu_{\sigma(i)}^{\omega_{i}}\right)\right\rangle$,

(3) $\operatorname{PFHHA}_{\gamma}\left(\alpha_{1}, \alpha_{2}, \ldots, \alpha_{n}\right)=\left\langle\left(\prod_{i=1}^{n}(1+(\gamma-1)\right.\right.$ $\left.\tilde{\mu}_{\sigma(i)}\right)^{\omega_{i}}-\prod_{i=1}^{n}\left(1-\widetilde{\mu}_{\sigma(i)}\right)^{\omega_{i}} / \prod_{i=1}^{n}\left(1+(\gamma-1) \widetilde{\mu}_{\sigma(i)}\right)^{\omega_{i}}+$ $\left.(\gamma-1) \prod_{i=1}^{n}\left(1-\tilde{\mu}_{\sigma(i)}\right)^{\omega_{i}}\right),\left(\gamma \prod_{i=1}^{n} \tilde{\eta}_{\sigma(i)}^{\omega_{i}} / \prod_{i=1}^{n}(1+(\gamma-\right.$ 1) $\left.\left.\left(1-\widetilde{\eta}_{\sigma(i)}\right)\right)^{\omega_{i}}+(\gamma-1) \prod_{i=1}^{n} \widetilde{\eta}_{\sigma(i)}^{\omega_{i}}\right),\left(\gamma \prod_{i=1}^{n} \widetilde{v}_{\sigma(i)}^{\omega_{i}} /\right.$ $\left.\left.\prod_{i=1}^{n}\left(1+(\gamma-1)\left(1-\widetilde{v}_{\sigma(i)}\right)\right)^{\omega_{i}}+(\gamma-1) \prod_{i=1}^{n} \widetilde{v}_{\sigma(i)}^{\omega_{i}}\right)\right\rangle$
Definition 13. (see [53]). Three PF weighted interaction aggregation operators are listed as follows:

(1) $\operatorname{PFWIA}\left(\alpha_{1}, \alpha_{2}, \ldots, \alpha_{n}\right)=\left\langle 1-\prod_{i=1}^{n}\left(1-\mu_{i}\right)^{\omega_{i}}\right.$, $\prod_{i=1}^{n}\left(1-\mu_{i}\right)^{\omega_{i}}-\prod_{i=1}^{n}\left(\nu_{i}+\pi_{i}\right)^{\omega_{i}}, \prod_{i=1}^{n}\left(\nu_{i}+\pi_{i}\right)^{\omega_{i}}-$ $\left.\prod_{i=1}^{n} \pi_{i}^{\omega_{i}}\right\rangle$

(2) $\operatorname{PFOWIA}\left(\alpha_{1}, \alpha_{2}, \ldots, \alpha_{n}\right)=\left\langle 1-\prod_{i=1}^{n}\left(1-\mu_{\sigma(i)}\right)^{\omega_{i}}\right.$, $\prod_{i=1}^{n}\left(1-\mu_{\sigma(i)}\right)^{\omega_{i}}-\prod_{i=1}^{n} \quad\left(\nu_{\sigma(i)}+\pi_{\sigma(i)}\right)^{\omega_{i}}$, $\left.\prod_{i=1}^{n}\left(\nu_{\sigma(i)}+\pi_{\sigma(i)}\right)^{\omega_{i}}-\prod_{i=1}^{n} \pi_{\sigma(i)}^{\omega_{i}}\right\rangle$,

(3) $\operatorname{PFHOWIA}\left(\alpha_{1}, \alpha_{2}, \ldots, \alpha_{n}\right)=\left\langle 1-\prod_{i=1}^{n}\left(1-\tilde{\mu}_{\sigma(i)}\right)^{\omega_{i}}\right.$, $\prod_{i=1}^{n}\left(1-\tilde{\mu}_{\sigma(i)}\right)^{\omega_{i}}-\prod_{i=1}^{n}\left(\widetilde{v}_{\sigma(i)}+\tilde{\pi}_{\sigma(i)}\right)^{\omega_{i}}$, $\left.\prod_{i=1}^{n}\left(\widetilde{\nu}_{\sigma(i)}+\widetilde{\pi}_{\sigma(i)}\right)^{\omega_{i}}-\prod_{i=1}^{n} \tilde{\pi}_{\sigma(i)}^{\omega_{i}}\right\rangle$.

Definition 14 (see [55]). Let $\Re \geq 1$. Two PF Dombi weighted averaging operators, which are provided by Jana et al., are listed as follows:

(1) $\operatorname{PFDWA}_{\mathfrak{R}}\left(\alpha_{1}, \alpha_{2}, \ldots, \alpha_{n}\right)=\left\langle 1-\left(1 / 1+\left[\sum_{i=1}^{n} \omega_{i}\right.\right.\right.$ $\left.\left.\left(\mu_{i} / 1-\mu_{i}\right)^{\Re}\right]^{1 / \Re}\right),\left(1 / 1+\left[\sum_{i=1}^{n} \omega_{i}\left(1-\eta_{i} / \eta_{i}\right)^{\Re{ }^{1 / R}}\right)\right.$, $\left.\left(1 / 1+\left[\sum_{i=1}^{n} \omega_{i}\left(1-\nu_{i} / \nu_{i}\right)^{\Re}\right]^{1 / R}\right)\right\rangle$,

(2) $\operatorname{PFDOWA}_{\mathfrak{R}}\left(\alpha_{1}, \alpha_{2}, \ldots, \alpha_{n}\right)=\left\langle 1-\left(1 / 1+\left[\sum_{i=1}^{n} \omega_{i}\right.\right.\right.$ $\left.\left.\left(\mu_{\sigma(i)} / 1-\mu_{\sigma(i)}\right)^{\Re}\right]^{1 / \Re}\right),\left(1 / 1+\left[\sum_{i=1}^{n} \omega_{i}\left(1-\eta_{\sigma(i)}\right.\right.\right.$ $\left.\left.\left.\left./ \eta_{\sigma(i)}\right)^{\Re}\right]^{1 / \Re}\right),\left(1 / 1+\left[\sum_{i=1}^{n} \omega_{i}\left(1-\nu_{\sigma(i)} / \nu_{\sigma(i)}\right)^{\Re}\right]^{1 / \Re}\right)\right\rangle$,

(3) $\operatorname{PFDHWA}_{\mathfrak{R}}\left(\alpha_{1}, \alpha_{2}, \ldots, \alpha_{n}\right)=\left\langle 1-\left(1 / 1+\left[\sum_{i=1}^{n} \omega_{i}\right.\right.\right.$ $\left.\left.\left(\widetilde{\mu}_{\sigma(i)} / 1-\tilde{\mu}_{\sigma(i)}\right)^{\Re}\right]^{1 / \Re}\right),\left(1 / 1+\left[\sum_{i=1}^{n} \omega_{i}\left(1-\widetilde{\eta}_{\sigma(i)}\right.\right.\right.$ $\left.\left.\left.\left./ \tilde{\eta}_{\sigma(i)}\right)^{\Re}\right]^{1 / \Re}\right),\left(1 / 1+\left[\sum_{i=1}^{n} \omega_{i}\left(1-\widetilde{\nu}_{\sigma(i)} / \widetilde{\nu}_{\sigma(i)}\right)^{\Re}\right]^{1 / \Re}\right)\right\rangle$.

Example 2. Let $U=\left\{x_{1}, x_{2}, x_{3}, x_{4}, x_{5}, x_{6}\right\} \quad$ and $\mathrm{C}=\left\{C_{1}, C_{2}, C_{3}, C_{4}, C_{5}, C_{6}\right\}$ is listed as follows:

$$
\begin{aligned}
& C_{1}=\left\{\left\langle x_{1}, 1,0,0\right\rangle,\left\langle x_{2}, 1,0,0\right\rangle,\left\langle x_{3}, 0.1,0.1,0.1\right\rangle,\left\langle x_{4}, 0.6,0.2,0.2\right\rangle,\left\langle x_{5}, 0.6,0.1,0.3\right\rangle,\left\langle x_{6}, 0.2,0.6,0.1\right\rangle\right\}, \\
& C_{2}=\left\{\left\langle x_{1}, 0.4,0.2,0.2\right\rangle,\left\langle x_{2}, 0.6,0.2,0.1\right\rangle,\left\langle x_{3}, 1,0,0\right\rangle,\left\langle x_{4}, 0.2,0.4,0.4\right\rangle,\left\langle x_{5}, 0.2,0.4,0.3\right\rangle,\left\langle x_{6}, 0.4,0.4,0.1\right\rangle\right\}, \\
& C_{3}=\left\{\left\langle x_{1}, 0.1,0.8,0.1\right\rangle,\left\langle x_{2}, 0.7,0.1,0.2\right\rangle,\left\langle x_{3}, 0.4,0.2,0.2\right\rangle,\left\langle x_{4}, 0.3,0.3,0.2\right\rangle,\left\langle x_{5}, 1,0,0\right\rangle,\left\langle x_{6}, 0.2,0.2,0.2\right\rangle\right\}, \\
& C_{4}=\left\{\left\langle x_{1}, 0.8,0.1,0.1\right\rangle,\left\langle x_{2}, 1,0,0\right\rangle,\left\langle x_{3}, 0.9,0.1,0\right\rangle,\left\langle x_{4}, 0.7,0.1,0.1\right\rangle,\left\langle x_{5}, 0.2,0.5,0.3\right\rangle,\left\langle x_{6}, 0.3,0.1,0\right\rangle\right\}, \\
& C_{5}=\left\{\left\langle x_{1}, 0.3,0.7,0\right\rangle,\left\langle x_{2}, 0.3,0.6,0.1\right\rangle,\left\langle x_{3}, 0.2,0.1,0.1\right\rangle,\left\langle x_{4}, 0.1,0.8,0.1\right\rangle,\left\langle x_{5}, 0.7,0.1,0.1\right\rangle,\left\langle x_{6}, 1,0,0\right\rangle\right\}, \\
& C_{6}=\left\{\left\langle x_{1}, 1,0,0\right\rangle,\left\langle x_{2}, 0.1,0.2,0.7\right\rangle,\left\langle x_{3}, 0.3,0.2,0.5\right\rangle,\left\langle x_{4}, 1,0,0\right\rangle,\left\langle x_{5}, 0.4,0.1,0.3\right\rangle,\left\langle x_{6}, 0.2,0.6,0.1\right\rangle\right\} .
\end{aligned}
$$

It is easy to see that for each $x \in U$, there exists $C \in \mathbf{C}$ such that $C(x)=1_{\mathbb{P}}$. Then, $\mathrm{C}$ is a PF covering, i.e., $\cup_{i=1}^{6} C_{i}=U$. By these methods in Definitions 10-14, the ranking results of six alternatives are shown in Table 1. At this point, regardless of the weight value, the ranking result remains unchanged. Accordingly, decision makers cannot choose the optimal alternative.

To solve the MCDM problems which cannot be solved by these methods [47-49, 53, 55, 56] effectively, the CPFRS model is proposed in this section.

\subsection{PF Neighborhood}

Definition 15. A PF neighborhood operator is a mapping $N: U \longrightarrow \mathscr{F}(U)$.

Definition 16. Let $\mathbf{C}$ be a PF covering and $x \in U$; then, the $\mathrm{PF}$ neighborhood system of $x$ is given by

$$
\mathfrak{L}(\mathbf{C}, x)=\left\{A \in \mathbf{C}: A(x) \neq 0_{\mathbb{P}}\right\} .
$$


TABLE 1: The ranking results of six alternatives by using different operators.

\begin{tabular}{|c|c|c|}
\hline Methods & $C_{1}(x), C_{2}(x), C_{3}(x), C_{4}(x), C_{5}(x), C_{6}(x)(x \in U)$ & The ranking results of six alternatives \\
\hline The PFWA operator in $[47,48]$ & $1_{\mathbb{P}}$ & $x_{1}=x_{2}=x_{3}=x_{4}=x_{5}=x_{6}$ \\
\hline The PFOWA operator in $[47,48]$ & $1_{\mathbb{P}}$ & $x_{1}=x_{2}=x_{3}=x_{4}=x_{5}=x_{6}$ \\
\hline The PFHWA operator in $[47,48]$ & $1_{\mathbb{P}}$ & $x_{1}=x_{2}=x_{3}=x_{4}=x_{5}=x_{6}$ \\
\hline The PFWA* operator in [49] & $1_{\mathbb{P}}$ & $x_{1}=x_{2}=x_{3}=x_{4}=x_{5}=x_{6}$ \\
\hline The PFOWA* operator in [49] & $1_{\mathbb{P}}$ & $x_{1}=x_{2}=x_{3}=x_{4}=x_{5}=x_{6}$ \\
\hline The PFHWA* operator in [49] & $1_{\mathbb{P}}$ & $x_{1}=x_{2}=x_{3}=x_{4}=x_{5}=x_{6}$ \\
\hline The $\mathrm{PFHWA}_{\gamma}$ operator in $[47,56]$ & $1_{\mathbb{P}}$ & $x_{1}=x_{2}=x_{3}=x_{4}=x_{5}=x_{6}$ \\
\hline The PFHOWA ${ }_{\gamma}$ operator in $[47,56]$ & $1_{\mathbb{P}}$ & $x_{1}=x_{2}=x_{3}=x_{4}=x_{5}=x_{6}$ \\
\hline The PFHHWA $\gamma$ operator in $[47,56]$ & $1_{\mathbb{P}}$ & $x_{1}=x_{2}=x_{3}=x_{4}=x_{5}=x_{6}$ \\
\hline The PFWIA operator in [53] & $1_{\mathbb{P}}$ & $x_{1}=x_{2}=x_{3}=x_{4}=x_{5}=x_{6}$ \\
\hline The PFOWIA operator in [53] & $1_{\mathbb{P}}$ & $x_{1}=x_{2}=x_{3}=x_{4}=x_{5}=x_{6}$ \\
\hline The PFHOWIA operator in [53] & $1_{\mathbb{P}}$ & $x_{1}=x_{2}=x_{3}=x_{4}=x_{5}=x_{6}$ \\
\hline The $\mathrm{PFDWA}_{\mathfrak{R}}$ operator in [55] & Cannot be calculated & $\times$ \\
\hline The PFDOWA ${ }_{\Re}$ operator in [55] & Cannot be calculated & $x$ \\
\hline The PFDHWA $A_{\Re}$ operator in [55] & Cannot be calculated & $x$ \\
\hline
\end{tabular}

Remark 1. By the concept of PF covering, for each $x \in U$, there exists a set $A \in \mathbf{C}$ such that $A(x)=1_{\mathbb{P}}$. Hence, $\mathfrak{Q}(\mathbf{C}, x) \neq \varnothing$ for each $x \in U$. The following conclusions are clearly established:

(1) If $\mathbf{C}$ is a fuzzy covering, then $\mathfrak{Q}(\mathbf{C}, x)$ is the fuzzy neighborhood system of $x$ defined in [73]

(2) If $\mathbf{C}$ is crisp, then $\mathfrak{Q}(\mathbf{C}, x)$ is crisp neighborhood system of $x$ defined in [74]

Definition 17. Let $\mathbf{C}$ be a PF covering and $x \in U$; then, the PF minimal description of $x$ is given by

$\operatorname{md}(\mathbf{C}, x)=\{A \in \mathfrak{L}(\mathbf{C}, x):(\forall S \in \mathfrak{L}(\mathbf{C}, x)) S(x)=A(x), S \subseteq A \Rightarrow S=A\}$.

Definition 18. Let $\mathbf{C}$ be a $\mathrm{PF}$ covering and let $\mathscr{I}$ be a $\mathrm{PF}$ border implicator which satisfies the confinement law; the PF neighborhood $N^{\mathrm{C}}(x)$ of $x$ is defined by

$$
N^{\mathrm{C}}(x)(y)=\inf _{A \in \mathbf{C}} \mathscr{I}(A(x), A(y)) .
$$

Note that PF implicator $\mathscr{I}$ satisfies the confinement law; then, $\quad N^{\mathrm{C}}(x)(x)=\inf _{A \in \mathbf{C}} \mathscr{I}(A(x), A(x))=1_{\mathbb{P}}$ for each $x \in U$. Here, we call that $N^{\mathrm{C}}$ is reflective.

Proposition 2. Let $\mathrm{C}$ be a finite PF covering and let $\mathscr{I}$ be a $P F$ border implicator which satisfies the confinement law. Then, for any $x, y \in U$, we have

$$
\begin{aligned}
\inf _{A \in \mathbf{C}} \mathscr{I}(A(x), A(y)) & =\inf _{A \in \mathfrak{Q}(\mathbf{C}, x)} \mathscr{I}(A(x), A(y)) \\
& =\inf _{A \in \operatorname{md}(\mathbf{C}, x)} \mathscr{I}(A(x), A(y)) .
\end{aligned}
$$

Proof. First note that if $A(x)=0_{\mathbb{P}}$ for some $A \in \mathbf{C}$, then by $\mathscr{I}\left(0_{\mathbb{P}}, 0_{\mathbb{P}}\right)=\mathscr{I}\left(0_{\mathbb{P}}, 1_{\mathbb{P}}\right)=1_{\mathbb{P}}$ and monotonicity of $\mathscr{I}$, we can obtain that

$$
\begin{aligned}
1_{\mathbb{P}} & =\mathscr{I}\left(0_{\mathbb{P}}, 0_{\mathbb{P}}\right) \leq_{\mathbb{P}} \mathscr{I}(A(x), A(y)) \\
& =\mathscr{I}\left(0_{\mathbb{P}}, A(y)\right) \leq_{\mathbb{P}} \mathscr{I}\left(0_{\mathbb{P}}, 1_{\mathbb{P}}\right)=1_{\mathbb{P}} .
\end{aligned}
$$

Hence,

$$
\begin{aligned}
\inf _{A \in \mathbf{C}} \mathscr{I}(A(x), A(y))= & \inf \left(\inf _{A \in \mathfrak{Q}(\mathbf{C}, x)} \mathscr{I}(A(x), A(y)),\right. \\
& \left.\inf _{A \in \mathbf{C} \backslash(\mathbf{C}, x)} \mathscr{I}(A(x), A(y))\right), \\
= & \inf _{A \in \mathfrak{Q}(\mathbf{C}, x)} \mathscr{I}(A(x), A(y)) .
\end{aligned}
$$

Furthermore, since md $(\mathbf{C}, x) \subseteq \mathfrak{L}(\mathbf{C}, x)$, we have

$$
\begin{array}{r}
\inf _{A \in \mathfrak{Q}(\mathbf{C}, x)} \mathscr{I}(A(x), A(y))=\inf \left(\inf _{A \in \operatorname{md}(\mathbf{C}, x)} \mathscr{I}(A(x), A(y)),\right. \\
\\
\left.\inf _{A \in \mathfrak{Q}(\mathbf{C}, x) \backslash \operatorname{md}(\mathbf{C}, x)} \mathscr{I}(A(x), A(y))\right) .
\end{array}
$$


If $A \in \mathfrak{Q}(\mathbf{C}, x) \backslash \mathrm{md}(\mathbf{C}, x)$, then by Definition 17, there exists $A^{\prime} \in \operatorname{md}(\mathbf{C}, x)$ such that $A^{\prime} \subseteq A$ and $A^{\prime}(x)=A(x)$. Therefore, for all $y \in U$,

$$
\mathscr{I}(A(x), A(y))=\mathscr{I}\left(A^{\prime}(x), A(y)\right) \geq_{\mathbb{P}} \mathscr{I}\left(A^{\prime}(x), A^{\prime}(y)\right) .
$$

It is immediate that

$$
\begin{aligned}
& \inf _{A \in \operatorname{md}(\mathbf{C}, x)} \mathscr{I}(A(x), A(y)) \\
& \leq_{\mathbb{P}} \inf _{A \in \mathfrak{R}(\mathbf{C}, x) \backslash \operatorname{md}(\mathbf{C}, x)} \mathscr{I}(A(x), A(y)),
\end{aligned}
$$

and so

$$
\inf _{A \in \mathfrak{Q}(\mathbf{C}, x)} \mathscr{I}(A(x), A(y))=\inf _{A \in \operatorname{md}(\mathbf{C}, x)} \mathscr{I}(A(x), A(y)) .
$$

Example 3. Consider the situation in Example 2, and let

$$
\mathscr{I}(x, y)=\mathscr{I}_{\mathcal{S}_{p}, N_{s}}^{*}(x, y)= \begin{cases}1_{\mathbb{P}}, & \text { if } x \leq_{\mathbb{P}} y, \\ \left\langle x_{3}+y_{1}-x_{3} y_{1},\left(1-x_{1}-x_{2}-x_{3}\right) y_{2}, x_{1} y_{3}\right\rangle, & \text { otherwise }\end{cases}
$$

for $x=\left\langle x_{1}, x_{2}, x_{3}\right\rangle, y \in\left\langle x_{1}, x_{2}, x_{3}\right\rangle \in \mathbb{P}$, where $\mathcal{S}_{P}$ and $\mathcal{N}_{s}$ are shown in Example 1. Then, by means of equation (18), the results of $N^{\mathrm{C}}\left(x_{i}\right)(i=1,2,3,4,5,6)$ are shown in Table 2.

\subsection{CPFRS Model}

Definition 19. Let $\mathbf{C}$ be a PF covering of a nonempty universe $U$; then, a pair $(U, \mathbf{C})$ is called a picture fuzzy covering approximation space (PFCAS). Next, let $\mathscr{T}$ be a PF strong $t$-norm and $\mathscr{I}$ be a PF border implicator such that it satisfies the confinement law; then, for each $A \in \mathscr{F}(U)$ and $x \in U$, the lower and upper approximations of $A$ are defined by

$$
\begin{aligned}
& \underline{\operatorname{apr}}_{N^{\mathrm{c}, \mathscr{F}}}(A)(x)=\inf _{y \in U} \mathscr{I}\left(N^{\mathrm{C}}(x)(y), A(y)\right), \\
& \overline{\operatorname{apr}}_{N^{\mathrm{c}}, \mathscr{T}}(A)(x)=\sup _{y \in U} \mathscr{T}\left(N^{\mathrm{C}}(x)(y), A(y)\right) .
\end{aligned}
$$

If $\operatorname{apr}_{N^{\mathrm{C}}}(A)=\overline{\operatorname{apr}}_{N^{\mathrm{C}}}(A), A$ is called $(\mathscr{I}, \mathscr{T})$-definable. Otherwise, $A$ is called covering-based $(\mathscr{I}, \mathscr{T})$-picture fuzzy rough set $((\mathscr{I}, \mathscr{T})$-CPFRS $)$.

Remark 2. From Definition 19, we can consider the following cases:

(1) When the PF covering $\mathbf{C}$ degenerates into the fuzzy covering $C^{\prime}$, it is easy to see that $\mathrm{PF}$ implicator $\mathscr{I}, \mathrm{PF}$ $t$-norm $\mathscr{T}$, and the PF neighborhood $N^{\mathrm{C}}$ degenerate into fuzzy implicator $\mathscr{I}^{\prime}$, fuzzy $t$-norm $\mathrm{T}^{\prime}$, and the fuzzy neighborhood $N^{C^{\prime}}$, respectively. Then, equations (27) and (28) can turn into the following form [62]:

$$
\begin{aligned}
& \underline{\operatorname{apr}}_{N{ }^{c^{\prime}}, \mathscr{I}^{\prime}}(A)(x)=\inf _{y \in U} \mathscr{I}^{\prime}\left(N^{C^{\prime}}(x)(y), A(y)\right), \\
& \overline{\operatorname{apr}}_{N^{c^{\prime}}, T^{\prime}}(A)(x)=\sup _{y \in U} \mathrm{~T}^{\prime}\left(N^{C^{\prime}}(x)(y), A(y)\right) .
\end{aligned}
$$

(2) When PF implicator $\mathscr{F}, \mathrm{PF} t$-norm, and the PF neighborhood $N^{\mathrm{C}}$ degenerate into fuzzy implicator $\mathscr{I}^{\prime}$, fuzzy $t$-norm $\mathrm{T}^{\prime}$, and fuzzy relation $\mathscr{R}$, respectively, then equations (27) and (28) can turn into the following form [75]:

$$
\begin{aligned}
& \underline{\operatorname{apr}}_{\mathscr{R}, \mathscr{F}^{\prime}}(A)(x)=\inf _{y \in U} \mathscr{I}^{\prime}(\mathscr{R}(x)(y), A(y)), \\
& \overline{\operatorname{apr}}_{\mathscr{R}, \mathrm{T}^{\prime}}(A)(x)=\sup _{y \in U} \mathrm{~T}^{\prime}(\mathscr{R}(x)(y), A(y)) .
\end{aligned}
$$

(3) When the PF covering $\mathbf{C}$ degenerates into the IF covering $C^{\prime \prime}$, it is easy to see that $\mathrm{PF}$ implicator $\mathcal{F}, \mathrm{PF}$ $t$-norm $\mathscr{T}$, and the PF neighborhood $N^{\mathrm{C}}$ degenerate into IF implicator $\mathscr{I}^{\prime \prime}$, IF $t$-norm $\mathrm{T}^{\prime \prime}$, and the IF neighborhood $N^{C^{\prime \prime}}$, respectively. Then, equations (27) and (28) can turn into the following form [66]:

$$
\begin{aligned}
& \underline{\operatorname{apr}}_{N C^{\prime \prime}, \mathscr{F}^{\prime \prime}}(A)(x)=\inf _{y \in U} \mathscr{I}^{\prime}\left(N^{C^{\prime \prime}}(x)(y), A(y)\right), \\
& \overline{\operatorname{apr}}_{N C^{\prime \prime}, T^{\prime \prime}}(A)(x)=\sup _{y \in U} T^{\prime}\left(N^{C^{\prime \prime}}(x)(y), A(y)\right) .
\end{aligned}
$$

(4) When PF implicator $\mathscr{I}$, PF $t$-norm $\mathscr{T}$, and the PF neighborhood $N^{\mathrm{C}}$ degenerate into IF implicator $\mathscr{I}^{\prime \prime}$, IF $t$-norm $\mathrm{T}^{\prime \prime}$, and IF relation $\rho$, respectively, then equations (27) and (28) can turn into the following form [76]:

$$
\begin{aligned}
& \operatorname{apr}_{\rho, \mathscr{F}^{\prime \prime}}(A)(x)=\inf _{y \in U} \mathscr{I}^{\prime \prime}(\mathscr{R}(x)(y), A(y)), \\
& \overline{\operatorname{apr}}_{\rho, \mathrm{T}^{\prime \prime}}(A)(x)=\sup _{y \in U} \mathrm{~T}^{\prime \prime}(\mathscr{R}(x)(y), A(y)) .
\end{aligned}
$$

(5) When the PF neighborhood $N^{\mathrm{C}}$ is replaced by a PF relation $\sigma$, then equations (27) and (28) can turn into the following form [36]: 
TABLe 2: The results of $N^{\mathrm{C}}\left(x_{i}\right)$.

\begin{tabular}{lcccccc}
\hline$N^{\mathrm{C}}\left(x_{i}\right) / U$ & $x_{1}$ & $x_{2}$ & $x_{3}$ & $x_{4}$ & $x_{5}$ & $x_{6}$ \\
\hline$N^{\mathrm{C}}\left(x_{1}\right)$ & $\langle 1,0,0\rangle$ & $\langle 0.3,0,0.7\rangle$ & $\langle 0.1,0.4,0.5\rangle$ & $\langle 0.36,0.44,0.2\rangle$ & $\langle 0.28,0.42,0.3\rangle$ & $\langle 0.2,0,0.1\rangle$ \\
$N^{\mathrm{C}}\left(x_{2}\right)$ & $\langle 0.28,0.6,0.12\rangle$ & $\langle 1,0,0\rangle$ & $\langle 0.1,0.76,0.14\rangle$ & $\langle 0.19,0.57,0.24\rangle$ & $\langle 0.2,0,0.3\rangle$ & $\langle 0.2,0.66,0.14\rangle$ \\
$N^{\mathrm{C}}\left(x_{3}\right)$ & $\langle 0.28,0.52,0.2\rangle$ & $\langle 0.55,0,0.21\rangle$ & $\langle 1,0,0\rangle$ & $\langle 0.19,0.41,0.4\rangle$ & $\langle 0.2,0,0.3\rangle$ & $\langle 0.3,0.6,0.1\rangle$ \\
$N^{\mathrm{C}}\left(x_{4}\right)$ & $\langle 0.28,0.16,0.03\rangle$ & $\langle 0.1,0,0.7\rangle$ & $\langle 0.28,0.22,0.5\rangle$ & $\langle 1,0,0\rangle$ & $\langle 0.28,0.42,0.3\rangle$ & $\langle 0.2,0,0.1\rangle$ \\
$N^{\mathrm{C}}\left(x_{5}\right)$ & $\langle 0.1,0,0.1\rangle$ & $\langle 0.37,0.04,0.28\rangle$ & $\langle 0.28,0.52,0.2\rangle$ & $\langle 0.19,0.61,0.2\rangle$ & $\langle 1,0,0\rangle$ & $\langle 0.2,0,0.2\rangle$ \\
$N^{\mathrm{C}}\left(x_{6}\right)$ & $\langle 0.28,0.64,0.08\rangle$ & $\langle 0.19,0.02,0.14\rangle$ & $\langle 0.19,0.71,0.1\rangle$ & $\langle 0.28,0.04,0.16\rangle$ & $\langle 0.2,0.08,0.12\rangle$ & $\langle 1,0,0\rangle$ \\
\hline
\end{tabular}

$$
\begin{aligned}
& \underline{\operatorname{apr}}_{\sigma, \mathscr{I}}(A)(x)=\inf _{y \in U} \mathscr{I}(\sigma(x)(y), A(y)), \\
& \overline{\operatorname{apr}}_{\sigma, \mathscr{T}}(A)(x)=\sup _{y \in U} \mathscr{T}(\sigma(x)(y), A(y)) .
\end{aligned}
$$

Example 4. Let PFCAS $(U, \mathrm{C})$ be listed as in Example 2, and let $\mathscr{I}=\mathscr{I}_{\mathcal{S}_{P}, \mathcal{N}_{s}}^{*}$ and $\mathscr{T}=\mathscr{T}_{I}$, where $\mathcal{S}_{P}, \mathcal{N}_{s}$, and $\mathscr{T}_{I}$ are shown in Example 1. Consider the PFS

$$
A=\left\{\left\langle x_{1}, 0.7,0.1,0.2\right\rangle,\left\langle x_{2}, 0.4,0.2,0\right\rangle,\left\langle x_{3}, 0.2,0,0.1\right\rangle,\left\langle x_{4}, 1,0,0\right\rangle,\left\langle x_{5}, 0.5,0,0.1\right\rangle,\left\langle x_{6}, 0.2,0.3,0.4\right\rangle\right\}
$$

By Definition 19 and Table 2, we have

$\underline{\operatorname{apr}}_{N^{\mathrm{C}}, \mathscr{F}}(A)=\left\{\left\langle x_{1}, 0.18,0.62,0.2\right\rangle,\left\langle x_{2}, 0.312,0,0.08\right\rangle,\left\langle x_{3}, 0.2,0,0.1\right\rangle,\left\langle x_{4}, 0.28,0.21,0.08\right\rangle,\left\langle x_{5}, 0.36,0.54,0.1\right\rangle,\left\langle x_{6}, 0.2,0,0.4\right\rangle\right\}$,

$\overline{\operatorname{apr}}_{N \mathrm{c}, \mathscr{T}}(A)=\left\{\left\langle x_{1}, 0.7,0.1,0.2\right\rangle,\left\langle x_{2}, 0.4,0.2,0\right\rangle,\left\langle x_{3}, 0.4,0,0.1\right\rangle,\left\langle x_{4}, 1,0,0\right\rangle,\left\langle x_{5}, 0.5,0,0.1\right\rangle,\left\langle x_{6}, 0.28,0,0.1\right\rangle\right\}$.

Proposition 3. For the operators $\underline{\operatorname{apr}}_{N^{\mathrm{C}}, \mathscr{I}}$ and $\overline{\operatorname{apr}}_{N^{\mathrm{C}}, \mathscr{T}}$, the following statements are true:

(1) $\underset{\operatorname{apr}_{N}^{\mathrm{c}, \mathscr{I}}}{ }(U)=\overline{\operatorname{apr}}_{N^{\mathrm{c}, \mathscr{T}}}(U)=U$

(2) $\operatorname{apr}_{N^{\mathrm{C}}, \mathscr{F}}(\varnothing)=\overline{\operatorname{apr}}_{N^{\mathrm{C}}, \mathscr{T}}(\varnothing)=\varnothing$

(3) $\overline{a p r}_{N^{\mathrm{C}}, \mathscr{I}}(A) \subseteq A \subseteq \overline{a p r}_{N^{\mathrm{C}}, \mathscr{T}}(A)$ for each $A \in \mathscr{F}(U)$

(4) For $A, B \in \mathscr{F}(U)$ such that $A \subseteq B$,

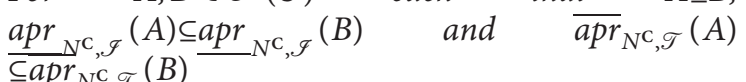

(5) $\overline{a p r}_{N^{\mathrm{c}}, \mathscr{T}}(A \cap B) \subseteq \overline{a p r}_{N^{\mathrm{c}}, \mathscr{T}}(A) \cap \overline{a p r}_{N^{\mathrm{c}}, \mathscr{T}}(B) \quad$ and $\underline{\operatorname{apr}}_{N^{\mathrm{C}, \mathscr{I}}}(A \cap B) \subseteq \underline{\operatorname{apr}}_{N^{\mathrm{C}}, \mathscr{I}}(A) \cap \underline{\operatorname{apr}}{ }_{N^{\mathrm{C}}, \mathscr{I}}(B)$ for each $A, B \in \mathscr{F}(U)$

Proof. (1) For each $x \in U$,

$$
\begin{aligned}
& \underline{\operatorname{apr}} N_{N^{\mathrm{C}}, \mathscr{T}}(U)(x)=\inf _{y \in U} \mathscr{I}\left(N^{\mathrm{C}}(x)(y), U(y)\right) \\
& \quad=\inf _{y \in U} \mathscr{I}\left(N^{\mathrm{C}}(x)(y), 1_{\mathbb{P}}\right) \geq_{\mathbb{P}} \inf _{y \in U} \mathscr{I}\left(1_{\mathbb{P}}, 1_{\mathbb{P}}\right) \\
& \quad=1_{\mathbb{P}}, \\
& \overline{\operatorname{apr}}_{N^{\mathrm{C}}, \mathscr{T}}(U)(x)=\sup _{y \in U}\left\{\mathscr{T}\left(N^{\mathrm{C}}(x)(y), U(y)\right)\right\} \\
& =\sup _{y \in U}\left\{\mathscr{T}\left(N^{\mathrm{C}}(x)(y), 1_{\mathbb{P}}\right)\right\} \\
& =\sup _{y \in U}\left\{N^{\mathrm{C}}(x)(y)\right\} \geq_{\mathbb{P}} N^{\mathrm{C}}(x)(x) \\
& =1_{\mathbb{P}} .
\end{aligned}
$$

Then, $\operatorname{apr}_{N^{\mathrm{C}}, \mathscr{G}}(U)(x)=\overline{\operatorname{apr}}_{N^{\mathrm{C}}, \mathscr{T}}(U)(x)=1_{\mathbb{P}}$ and so (1) holds.
(2) For each $x \in U$,

$$
\begin{aligned}
& \operatorname{apr}_{N^{\mathrm{c}}, \mathscr{I}}(\varnothing)(x)=\inf _{y \in U} \mathscr{I}\left(N^{\mathrm{C}}(x)(y), \varnothing(y)\right) \\
& =\inf _{y \in U} \mathscr{I}\left(N^{\mathrm{C}}(x)(y), 0_{\mathbb{P}}\right) \leq_{\mathbb{P}} \mathscr{I}\left(N^{\mathrm{C}}(x)(x), 0_{\mathbb{P}}\right) \\
& =\mathscr{I}\left(1_{\mathbb{P}}, 0_{\mathbb{P}}\right) \\
& =0_{\mathbb{P}} \text {, } \\
& \overline{\operatorname{apr}}_{N^{\mathrm{C}}, \mathscr{T}}(\varnothing)(x)=\sup _{y \in U}\left\{\mathscr{T}\left(N^{\mathrm{C}}(x)(y), \varnothing(y)\right)\right\} \\
& =\sup _{y \in U}\left\{\mathscr{T}\left(N^{\mathrm{C}}(x)(y), 0_{\mathbb{P}}\right)\right\} \leq_{\mathbb{P}} \sup _{y \in U}\left\{\mathscr{T}\left(1_{\mathbb{P}}, 0_{\mathbb{P}}\right)\right\} \\
& =\sup _{y \in U}\left\{0_{\mathbb{P}}\right\} \\
& =0_{\mathbb{P}} \text {. }
\end{aligned}
$$

Then, apr $N_{N^{\mathrm{C}}}(\varnothing)(x)=\overline{\operatorname{apr}}_{N^{\mathrm{c}}, \mathscr{T}}(\varnothing)(x)=0_{\mathbb{P}}$ and so (2) holds.

(3) For each $x \in U, A \in \mathscr{F}(U)$,

$$
\begin{aligned}
& \frac{\operatorname{apr}}{N^{\mathrm{C}}, \mathscr{F}}(A)(x)=\inf _{y \in U} \mathscr{I}\left(N^{\mathrm{C}}(x)(y), A(y)\right) \\
& \quad \leq_{\mathbb{P}} \mathscr{I}\left(N^{\mathrm{C}}(x)(x), A(x)\right) \\
& \quad=\mathscr{I}\left(1_{\mathbb{P}}, A(x)\right) \leq_{\mathbb{P}} A(x), \\
& \overline{\operatorname{apr}}_{N^{\mathrm{C}}, \mathscr{T}}(A)(x)=\sup _{y \in U}\left\{\mathscr{T}\left(N^{\mathrm{C}}(x)(y), A(y)\right)\right\} \\
& \quad \geq_{\mathbb{P}} \mathscr{T}\left(N^{\mathrm{C}}(x)(x), A(x)\right) \\
& =\mathscr{T}\left(1_{\mathbb{P}}, A(x)\right) \\
& =A(x) .
\end{aligned}
$$


Then, $\underline{\operatorname{apr}}_{N^{\mathrm{c}, \mathscr{I}}}(A) \subseteq A \subseteq \overline{\operatorname{apr}}_{N^{\mathrm{c}}, \mathscr{T}}(A)$.

(4) Let $A, B \in \mathscr{F}(U)$ such that $A \subseteq B$; then, $A(x) \leq_{\mathbb{P}} B(x)$ for each $x \in U$. By the monotonicity of $\mathscr{F}$, we can prove that $\underline{\operatorname{apr}}_{N^{\mathrm{c}}, \mathscr{F}}(A) \subseteq \underline{\operatorname{apr}}_{N^{\mathrm{c}}, \mathscr{F}}(B)$ and $\overline{\operatorname{apr}}_{N \mathrm{c}, \mathscr{T}}(A) \subseteq \overline{\operatorname{apr}}_{N \mathrm{c}, \mathscr{T}}(B)$.

(5) From (1) and (3) of Definition 2, we have $A \cap B \subseteq A$ and $A \cap B \subseteq B$ for each $A, B \in \mathscr{F}(U)$. It is easy to obtain (5) by (4).

\section{An Approach to MCDM with PF Information Based on CPFRS Model}

4.1. Decision-Making Background. To accelerate the development of urbanization in China, the number of buildings is rapidly increasing. At the same time, China is also facing increasingly prominent resource constraints. To maximize the use of resources and protect the environment, in recent years, the Ministry of Construction of China has proposed "building a resource-saving and environment-friendly society" as its guiding principle to promote the modernization of the green building industry. Green buildings have the three characteristics of energy-saving, resource-saving, and returning to nature, namely, saving resources to the greatest extent, protecting the environment, and reducing pollution. Compared with ordinary buildings, the characteristics of green buildings make developers face project investment risks. To ensure the smooth progress of the project, the risk management of the green building project by the project management team is of great significance. By accessing relevant data and consulting experts, some characteristics of the risk (e.g., the possibility of occurrence, influence, and unpredictability) can be obtained. However, the resources for risk management are limited and risk factors are often numerous. Therefore, the project management team uses risk assessment to determine the priority of risk management which can greatly improve the efficiency of risk management. This is a MCDM problem in the field of risk management. Since most of the data obtained are vague or uncertain, green building is a process involving uncertainty and vague risk. Using traditional methods, we cannot effectively make risk management decisions. However, our proposed method can help the risk management team to do this work.

Let $U=\left\{a_{1}, a_{2}, \ldots, a_{n}\right\}$ be composed of $n$ risk factors and $\mathbf{C}=\left\{C_{1}, C_{2}, \ldots, C_{m}\right\}$ be composed of $m$ criteria. The evaluation value of option $a_{i}$ under criteria $C_{j}$ is expressed by the PFN $x_{i j}=\left\langle\mu_{i j}, \eta_{i j}, v_{i j}\right\rangle$, where $\mu_{i j}, \eta_{i j}$, and $v_{i j}$ indicate the positive membership degree, neutral membership degree, and negative membership degree that the risk factor $a_{i}$ satisfies the criteria $C_{j}$ given by a lots of experts' evaluation, respectively. For example, a 10member expert group evaluates risk factor $a_{1}$ as $\langle 0.2,0.3,0.4\rangle$ under criteria $C_{1}$; then, evaluation value of risk factor $a_{1}$ under criteria $C_{1}$ is denoted by $x_{11}=\left\langle\mu_{11}, \eta_{11}, v_{11}\right\rangle=\langle 0.2,0.3,0.4\rangle$, where $\mu_{11}=0.2$ means that two experts strongly believe that risk factor $a_{1}$ satisfies the criteria $C_{1} ; \eta_{11}=0.3$ means that three experts are neutral on the risk factor $a_{1}$ satisfying the criteria $C_{1}$; $v_{11}=0.4$ means that four experts firmly believe that the risk factor $a_{1}$ does not satisfy the criteria $C_{1}$; and $\pi_{11}=$ $1-\mu_{11}-\eta_{11}-v_{11}=0.1$ means that one expert refused to state whether risk factor $a_{1}$ satisfies the criteria $C_{1}$. Suppose that for each risk factor $a \in U$, there exist criteria $C \in \mathbf{C}$ such that the value of $a$ under the criteria $C$ is equal to $1_{\mathbb{P}}$. Obviously, $\mathrm{C}$ is a PF covering of $U$.

4.2. Decision-Making Methodology. Firstly, by many authoritative experts, a risk evaluation matrix $M=\left[x_{i j}\right]_{n \times m}$ is given as in Table 3.

Here, we consider two types of criteria in MCDM, namely, cost type and benefit type. Normally, the cost-type criteria should be transformed into corresponding benefittype criteria. So, we transform the risk evaluation matrix $M=\left[x_{i j}\right]_{n \times m}$ into a normalized decision matrix $M^{*}=\left[x_{i j}^{*}\right]_{n \times m}$, where

$$
x_{i j}^{*}= \begin{cases}x_{i j}, & \text { if } C_{j} \text { is a benefit }- \text { type criterion }, \\ \bar{x}_{i j}, & \text { if } C_{j} \text { is a cost }- \text { type criterion }\end{cases}
$$

for $i=1,2, \ldots, n$ and $j=1,2, \ldots, m$.

Then, the positive ideal solution and the negative ideal solution are obtained by the following equations:

$$
\begin{aligned}
& I^{+}=\left\{\left\langle a, \mu_{a}, \eta_{a}, v_{a}\right\rangle:(a \in U)\left\langle\mu_{a}, \eta_{a}, v_{a}\right\rangle=\sup _{C \in \mathbf{C}} C(a)\right\}, \\
& I^{-}=\left\{\left\langle a, \mu_{a}, \eta_{a}, v_{a}\right\rangle:(a \in U)\left\langle\mu_{a}, \eta_{a}, v_{a}\right\rangle=\inf _{C \in \mathbf{C}} C(a)\right\} .
\end{aligned}
$$

Furthermore, by the score function,

$$
L(\alpha)=\frac{\mu_{\alpha}-v_{\alpha}}{1-\eta_{\alpha}}, \quad \eta_{\alpha} \neq 1,
$$

and the algebraic product $\delta_{P}$; for each $a_{i} \in U$, two ranking functions of $a_{i}$ are defined as

$$
\begin{aligned}
& S^{+}\left(a_{i}\right)=L\left(\mathcal{S}_{P}\left(\underline{\operatorname{apr}}_{N^{\mathrm{c}, \mathscr{F}}}\left(I^{+}\right)\left(a_{i}\right), \overline{\operatorname{apr}}_{N^{\mathrm{c}}, \mathscr{T}}\left(I^{+}\right)\left(a_{i}\right)\right)\right), \\
& S^{-}\left(a_{i}\right)=L\left(\mathcal{S}_{P}\left(\underline{\operatorname{apr}}_{N^{\mathrm{c}, \mathscr{T}}}\left(I^{-}\right)\left(a_{i}\right), \overline{\operatorname{apr}}_{N^{\mathrm{c}}, \mathscr{T}}\left(I^{-}\right)\left(a_{i}\right)\right)\right) .
\end{aligned}
$$

Finally, based on the principle of the TOPSIS methods, the intimate function is obtained as follows:

$$
\delta\left(a_{i}\right)=\frac{S^{-}\left(a_{i}\right)}{S^{-}\left(a_{i}\right)+S^{+}\left(a_{i}\right)}, \quad a_{i} \in U .
$$

We can rank these risk factors and choose the highest risk factor by the following ranking rule: 
TABLE 3: Risk evaluation matrix.

\begin{tabular}{ccccc}
\hline$U / \mathbf{C}$ & $C_{1}$ & $C_{2}$ & $\cdots$ & $C_{m}$ \\
\hline$a_{1}$ & $x_{11}$ & $x_{12}$ & $\cdots$ & $x_{1 m}$ \\
$a_{2}$ & $x_{21}$ & $x_{22}$ & $\cdots$ & $x_{2 m}$ \\
$\vdots$ & $\vdots$ & $\vdots$ & $\vdots$ & $\vdots$ \\
$a_{n}$ & $x_{n 1}$ & $x_{n 2}$ & $\cdots$ & $x_{n m}$ \\
\hline
\end{tabular}

$$
\delta\left(a_{i}\right)>\delta\left(a_{j}\right) \Rightarrow a_{i}>a_{j}
$$

4.3. Procedure for Decision-Making Method. An algorithm of the proposed method is shown as follows:

Input: risk evaluation matrix $M=\left[x_{i j}\right]_{n \times m}$.

Output: a ranking result of all risk factors.

Step 1: by equation (39), normalized decision matrix $M^{*}=\left[x_{i j}^{*}\right]_{n \times m}$ is obtained.

Step 2: by equations (40) and (41), compute the positive ideal solution $I^{+}$and the negative ideal solution $I^{-}$.

Step 3: by equations (27) and (28), compute a pair of approximation operators $\operatorname{apr}_{N^{\mathrm{C}, \mathscr{I}}}$ and $\overline{\operatorname{apr}}_{N^{\mathrm{C}}, \mathscr{T}}$ of $I^{+}$ and $I^{-}$

Step 4: by equations (42)-(44), compute two ranking functions $S^{+}\left(a_{i}\right)$ and $S^{-}\left(a_{i}\right)$ for each $a_{i} \in U$.
Step 5: by equation (45), compute intimate function $\delta\left(a_{i}\right)$ for each $a_{i} \in U$.

Step 6: according to rule (46), rank these risk factors and choose the highest risk factor.

4.4. An Example. In a construction company, seven key risk factors, which are based on the root causes of risks in the green building project, are adopted by consulting the relevant data and experts. The seven risk factors are denoted by $U=\left\{a_{1}, a_{2}, a_{3}, a_{4}, a_{5}, a_{6}, a_{7}\right\}$, where $a_{1}, a_{2}, a_{3}, a_{4}, a_{5}, a_{6}$, and $a_{7}$ represent policy risk, market risk, manage risk, quality risk, period risk, security risk, and claim risk, respectively. For more information on the seven risk factors, see [3]. Meanwhile, the following four criteria $[2,3]$ are considered to evaluate the risk factors: probability of risk occurrence $\left(C_{1}\right)$, risk influence $\left(C_{2}\right)$, risk unpredictability $\left(C_{3}\right)$, and risk urgency $\left(C_{4}\right)$. Suppose that for each risk $a_{i}$, there at least exists a criterion $C_{j}$ such that $C_{j}\left(a_{i}\right)=1_{\mathbb{P}}$. Table 4 presents the project risk evaluation matrix under the $\mathrm{PF}$ environment by a lot of experts' diagnosis.

Firstly, from [3], we can know that the four criteria are all benefit type in the risk evaluation problem. Thus, the normalized decision matrix is the same as Table 4.

Secondly, by equations (40) and (41), the value of positive and negative ideal solutions is listed as

$$
\begin{aligned}
& I^{+}=\left\{\left\langle a_{1}, 1,0,0\right\rangle,\left\langle a_{2}, 1,0,0\right\rangle,\left\langle a_{3}, 1,0,0\right\rangle,\left\langle a_{4}, 1,0,0\right\rangle,\left\langle a_{5}, 1,0,0\right\rangle,\left\langle a_{6}, 1,0,0\right\rangle,\left\langle a_{7}, 1,0,0\right\rangle\right\}, \\
& I^{-}=\left\{\begin{array}{c}
\left\langle a_{1}, 0.3,0.4,0.3\right\rangle,\left\langle a_{2}, 0.2,0.6,0.2\right\rangle,\left\langle a_{3}, 0.3,0.3,0.4\right\rangle,\left\langle a_{4}, 0.2,0.3,0.5\right\rangle, \\
\left\langle a_{5}, 0.3,0.1,0.4\right\rangle,\left\langle a_{6}, 0.6,0.1,0.2\right\rangle,\left\langle a_{7}, 0.4,0.1,0.3\right\rangle
\end{array}\right\} .
\end{aligned}
$$

Here, suppose that $\mathscr{I}=\mathscr{I}_{\mathcal{S}_{p}, \mathcal{N}_{s}}^{*}$ and $\mathscr{T}=\mathscr{T}_{I}$; by means of equation (18), the result is listed as in Table 5.

Then, by equations (27) and (28), we have the following results:

$$
\begin{aligned}
\underline{\operatorname{apr}}_{N^{\mathrm{c}}, \mathscr{F}}\left(I^{+}\right)= & \overline{\operatorname{apr}}_{N^{\mathrm{c}}, \mathscr{T}}\left(I^{+}\right)=\left\{\left\langle a_{1}, 1,0,0\right\rangle,\left\langle a_{2}, 1,0,0\right\rangle,\left\langle a_{3}, 1,0,0\right\rangle,\left\langle a_{4}, 1,0,0\right\rangle,\left\langle a_{5}, 1,0,0\right\rangle,\left\langle a_{6}, 1,0,0\right\rangle,\left\langle a_{7}, 1,0,0\right\rangle\right\}, \\
\underline{\operatorname{apr}}_{N^{\mathrm{c}}, \mathscr{I}}\left(I^{-}\right)= & \left\{\left\langle a_{1}, 0.3,0,0.3\right\rangle,\left\langle a_{2}, 0.2,0,0.2\right\rangle,\left\langle a_{3}, 0.3,0,0.4\right\rangle,\left\langle a_{4}, 0.2,0,0.5\right\rangle,\left\langle a_{5}, 0.3,0.056,0.4\right\rangle,\left\langle a_{6}, 0.447,0.353,0.2\right\rangle,\right. \\
& \left.\left\langle a_{7}, 0.28,0.42,0.3\right\rangle\right\}, \\
\overline{\operatorname{apr}}_{N^{\mathrm{C}}, \mathscr{T}}\left(I^{-}\right)= & \left\{\left\langle a_{1}, 0.6,0,0.2\right\rangle,\left\langle a_{2}, 0.6,0.1,0.2\right\rangle,\left\langle a_{3}, 0.6,0.1,0.2\right\rangle,\left\langle a_{4}, 0.6,0.1,0.2\right\rangle,\left\langle a_{5}, 0.6,0,0.2\right\rangle,\left\langle a_{6}, 0.6,0.1,0.2\right\rangle,\right. \\
& \left.\left\langle a_{7}, 0.6,0.1,0.2\right\rangle\right\} .
\end{aligned}
$$

Furthermore, by equations (42)-(45), the results are listed as in Table 6.
According to rule (46), we rank seven risk factors as follows: 
TABLE 4: Risk evaluation matrix of the green building construction project.

\begin{tabular}{ccccc}
\hline & $C_{1}$ & $C_{2}$ & $C_{3}$ & $C_{4}$ \\
\hline$a_{1}$ & $\langle 0.6,0.1,0.2\rangle$ & $\langle 1,0,0\rangle$ & $\langle 0.3,0.1,0.1\rangle$ & $\langle 0.4,0.1,0.3\rangle$ \\
$a_{2}$ & $\langle 1,0,0\rangle$ & $\langle 0.5,0.2,0.2\rangle$ & $\langle 0.7,0.1,0.2\rangle$ & $\langle 0.2,0.4,0.1\rangle$ \\
$a_{3}$ & $\langle 0.3,0.3,0.4\rangle$ & $\langle 0.5,0.4,0.1\rangle$ & $\langle 1,0,0\rangle$ & $\langle 0.5,0.2,0.2\rangle$ \\
$a_{4}$ & $\langle 1,0,0\rangle$ & $\langle 0.8,0.1,0.1\rangle$ & $\langle 0.2,0.3,0.5\rangle$ & $\langle 0.2,0.5,0.2\rangle$ \\
$a_{5}$ & $\langle 0.7,0.2,0.1\rangle$ & $\langle 1,0,0\rangle$ & $\langle 0.3,0.1,0.4\rangle$ & $\langle 0.6,0.3,0.1\rangle$ \\
$a_{6}$ & $\langle 0.9,0.1,0\rangle$ & $\langle 0.6,0.1,0.2\rangle$ & $\langle 1,0,0\rangle$ & $\langle 0.7,0.1,0\rangle$ \\
$a_{7}$ & $\langle 0.8,0.1,0.1\rangle$ & $\langle 0.5,0.2,0.3\rangle$ & $\langle 0.4,0.1,0.3\rangle$ & $\langle 1,0,0\rangle$ \\
\hline
\end{tabular}

TABLE 5: The description for $N^{\mathrm{C}}\left(a_{i}\right)$.

\begin{tabular}{lccccccc}
\hline & $a_{1}$ & $a_{2}$ & $a_{3}$ & $a_{4}$ & $a_{5}$ & $a_{6}$ & $a_{7}$ \\
\hline$N^{\mathrm{C}}\left(a_{1}\right)$ & $\langle 1,0,0\rangle$ & $\langle 0.44,0.36,0.2\rangle$ & $\langle 0.44,0.03,0.24\rangle$ & $\langle 0.28,0.15,0.15\rangle$ & $\langle 0.37,0.05,0.12\rangle$ & $\langle 0.6,0,0.2\rangle$ & $\langle 0.46,0.24,0.3\rangle$ \\
$N^{\mathrm{C}}\left(a_{2}\right)$ & $\langle 0.44,0.36,0.2\rangle$ & $\langle 1,0,0\rangle$ & $\langle 0.3,0,0.4\rangle$ & $\langle 0.28,0.37,0.35\rangle$ & $\langle 0.44,0,0.28\rangle$ & $\langle 0.9,0,0\rangle$ & $\langle 0.52,0,0.21\rangle$ \\
$N^{\mathrm{C}}\left(a_{3}\right)$ & $\langle 0.3,0.55,0.15\rangle$ & $\langle 0.36,0.44,0.2\rangle$ & $\langle 1,0,0\rangle$ & $\langle 0.2,0,0.5\rangle$ & $\langle 0.3,0,0.4\rangle$ & $\langle 0.64,0,0.1\rangle$ & $\langle 0.4,0,0.3\rangle$ \\
$N^{\mathrm{C}}\left(a_{4}\right)$ & $\langle 0.52,0.28,0.2\rangle$ & $\langle 0.55,0,0.16\rangle$ & $\langle 0.3,0,0.4\rangle$ & $\langle 1,0,0\rangle$ & $\langle 0.7,0,0.1\rangle$ & $\langle 0.64,0,0.16\rangle$ & $\langle 0.55,0,0.24\rangle$ \\
$N^{\mathrm{C}}\left(a_{5}\right)$ & $\langle 0.46,0,0.18\rangle$ & $\langle 0.5,0,0.2\rangle$ & $\langle 0.37,0,0.28\rangle$ & $\langle 0.28,0.57,0.15\rangle$ & $\langle 1,0,0\rangle$ & $\langle 0.6,0,0.2\rangle$ & $\langle 0.5,0,0.3\rangle$ \\
$N^{\mathrm{C}}\left(a_{6}\right)$ & $\langle 0.3,0.49,0.21\rangle$ & $\langle 0.2,0.6,0.2\rangle$ & $\langle 0.3,0,0.36\rangle$ & $\langle 0.2,0,0.5\rangle$ & $\langle 0.3,0,0.4\rangle$ & $\langle 1,0,0\rangle$ & $\langle 0.4,0,0.3\rangle$ \\
$N^{\mathrm{C}}\left(a_{7}\right)$ & $\langle 0.4,0,0.3\rangle$ & $\langle 0.2,0,0.1\rangle$ & $\langle 0.37,0,0.32\rangle$ & $\langle 0.2,0,0.2\rangle$ & $\langle 0.51,0.02,0.16\rangle$ & $\langle 0.7,0,0\rangle$ & $\langle 1,0,0\rangle$ \\
\hline
\end{tabular}

TABLE 6: The description of $S^{+}\left(a_{i}\right), S^{-}\left(a_{i}\right)$, and $\delta\left(a_{i}\right)$.

\begin{tabular}{lccr}
\hline & $S^{+}\left(a_{i}\right)$ & $S^{-}\left(a_{i}\right)$ & $\delta\left(a_{i}\right)$ \\
\hline$a_{1}$ & 1 & 0.66 & 0.3976 \\
$a_{2}$ & 1 & 0.64 & 0.3902 \\
$a_{3}$ & 1 & 0.64 & 0.3902 \\
$a_{4}$ & 1 & 0.58 & 0.3671 \\
$a_{5}$ & 1 & 0.64 & 0.3902 \\
$a_{6}$ & 1 & 0.7658 & 0.4337 \\
$a_{7}$ & 1 & 0.7056 & 0.4137 \\
\hline
\end{tabular}

TABLE 7: Decision results obtained by applying different logical operators.

\begin{tabular}{lc}
\hline Three pairs of logic operators & The ranking results of seven risk factors \\
\hline $\mathscr{I}=\mathscr{I}_{\mathcal{S}_{M}, N_{s}}^{*}, \mathscr{T}=\mathscr{T}_{I}$ & $a_{6}>a_{7}=a_{2}>a_{5}=a_{3}>a_{1}>a_{4}$ \\
$\mathscr{I}=\mathscr{I}_{\mathcal{S}_{P}, \mathcal{N}_{s}}^{*}=\mathscr{T}_{I}$ & $a_{6}>a_{7}>a_{1}>a_{5}=a_{2}=a_{3}>a_{4}$ \\
$\mathscr{I}=\mathscr{I}_{\mathcal{S}_{L}, \mathcal{N}_{s}}^{*}, \mathscr{T}=\mathscr{T}_{I}$ & $a_{6}>a_{7}>a_{1}>a_{5}=a_{2}=a_{3}>a_{4}$ \\
\hline
\end{tabular}

$$
a_{6}>a_{7}>a_{1}>a_{5}=a_{2}=a_{3}>a_{4} .
$$

Obviously, decision makers can select the highest risk factor $a_{6}$ (security risk). In addition, other logical operators can be also considered in our proposed method. The ranking results of risk factors by using different logical operators are shown in Table 7 . It can be seen from Table 7 that the highest risk factor obtained by using different logical operators is the same in the ranking results.

4.5. Comparative Analysis. This section aims at verifying the effectiveness of the proposed method by comparing it with several classical methods. These comparative methods are shown as follows:
(1) The first type of comparative method is some aggregation operators in [47-49, 53-56]

(2) Considering the VIKOR method is one of the most widely used tools in MCDM methods, the second comparative method is VIKOR method based on picture fuzzy normalized projection (PFNP) model proposed by Wang et al. [3]

(3) The third comparative method is the approach based on picture fuzzy Hamming distance (PFHD) measure $[3,23]$

In order to make a comparative analysis among the results of our proposed method and the methods in $[3,23,47-49,53-56]$, the results of these methods are shown as in Table 8 and Figures $1-3$. Through Table 8 and Figures 1-3, we make a brief conclusion as follows: 
TABLE 8: Decision results obtained by applying different methods.

\begin{tabular}{|c|c|c|}
\hline References & Methods & The ranking results of seven risk factors \\
\hline Garg [47] and Wei [48] & $\begin{array}{l}\text { The PFWA operator } \\
\text { The PFOWA operator } \\
\text { The PFHWA operator }\end{array}$ & $\begin{array}{l}a_{1}=a_{2}=a_{3}=a_{4}=a_{5}=a_{6}=a_{7} \\
a_{1}=a_{2}=a_{3}=a_{4}=a_{5}=a_{6}=a_{7} \\
a_{1}=a_{2}=a_{3}=a_{4}=a_{5}=a_{6}=a_{7}\end{array}$ \\
\hline Wang [49] & $\begin{array}{l}\text { The PFWA* operator } \\
\text { The PFOWA* operator } \\
\text { The PFHWA* operator }\end{array}$ & $\begin{array}{l}a_{1}=a_{2}=a_{3}=a_{4}=a_{5}=a_{6}=a_{7} \\
a_{1}=a_{2}=a_{3}=a_{4}=a_{5}=a_{6}=a_{7} \\
a_{1}=a_{2}=a_{3}=a_{4}=a_{5}=a_{6}=a_{7}\end{array}$ \\
\hline Garg [47] and Wei [56] & $\begin{array}{l}\text { The } \mathrm{PFHWA}_{\gamma} \text { operator } \\
\text { The PFHOWA }{ }_{\gamma} \text { operator } \\
\text { The PFHHWA } \text { Pperator }^{\text {PFH }}\end{array}$ & $\begin{array}{l}a_{1}=a_{2}=a_{3}=a_{4}=a_{5}=a_{6}=a_{7} \\
a_{1}=a_{2}=a_{3}=a_{4}=a_{5}=a_{6}=a_{7} \\
a_{1}=a_{2}=a_{3}=a_{4}=a_{5}=a_{6}=a_{7}\end{array}$ \\
\hline Li et al. [53] & $\begin{array}{l}\text { The PFWIA operator } \\
\text { The PFOWIA operator } \\
\text { The PFHOWIA operator }\end{array}$ & $\begin{array}{l}a_{1}=a_{2}=a_{3}=a_{4}=a_{5}=a_{6}=a_{7} \\
a_{1}=a_{2}=a_{3}=a_{4}=a_{5}=a_{6}=a_{7} \\
a_{1}=a_{2}=a_{3}=a_{4}=a_{5}=a_{6}=a_{7}\end{array}$ \\
\hline Jana et al. [55] & $\begin{array}{l}\text { The PFDWA } \mathrm{PA}_{\mathfrak{R}} \text { operator } \\
\text { The PFDOWA } A_{\mathfrak{R}} \text { operator } \\
\text { The PFDHWA } \mathrm{P}_{\mathfrak{R}} \text { operator }\end{array}$ & $\begin{array}{l}\times \\
\times \\
\times \\
\end{array}$ \\
\hline Wang et al. [3] & VIKOR method based on PFNP model & $a_{6}>a_{3}>a_{5}>a_{7}>a_{1}>a_{2}>a_{4}$ \\
\hline Ju et al. [54] & The PFWIG operator & $a_{6}>a_{1}>a_{7}>a_{2}>a_{5}>a_{3}>a_{4}$ \\
\hline Wang et al. [3] and Coung [23] & PFHD measure & $a_{6}>a_{7}>a_{1}=a_{5}>a_{2}>a_{3}>a_{4}$ \\
\hline This paper & $\begin{array}{l}\text { Case } 1\left(\mathscr{I}=\mathscr{I}_{\mathcal{S}_{M}, \mathcal{N}_{s}}^{*} \text { and } \mathscr{T}=\mathscr{T}_{I} \text { in CPFRS model }\right) \\
\text { Case } 2\left(\mathscr{I}=\mathscr{I}_{\mathcal{S}_{P}, N_{s}}^{*} \text { and } \mathscr{T}=\mathscr{T}_{I} \text { in CPFRS model }\right) \\
\text { Case } 3\left(\mathscr{T}=\mathscr{I}_{\mathcal{S}_{L}, \mathcal{N}_{s}}^{*} \text { and } \mathscr{T}=\mathscr{T}_{I} \text { in CPFRS model }\right)\end{array}$ & $\begin{array}{l}a_{6}>a_{7}=a_{2}>a_{5}=a_{3}>a_{1}>a_{4} \\
a_{6}>a_{7}>a_{1}>a_{5}=a_{2}=a_{3}>a_{4} \\
a_{6}>a_{7}>a_{1}>a_{5}=a_{2}=a_{3}>a_{4}\end{array}$ \\
\hline
\end{tabular}

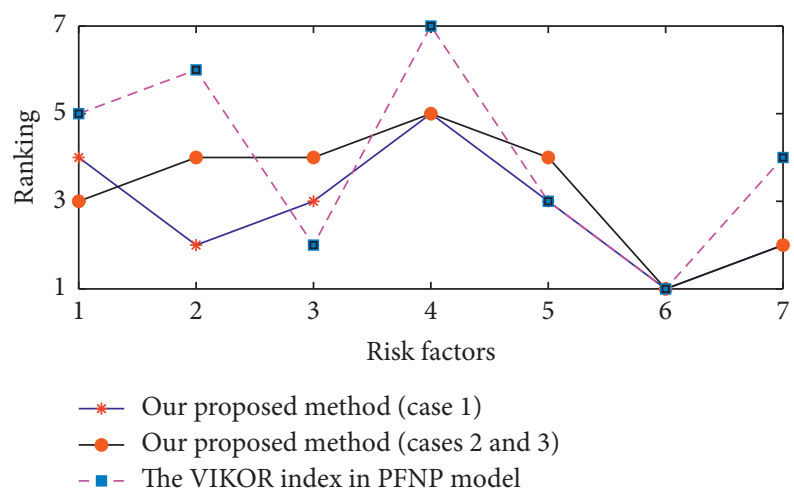

FIGURE 1: The comparison among the results of our proposed method (three cases) and PFNP model.

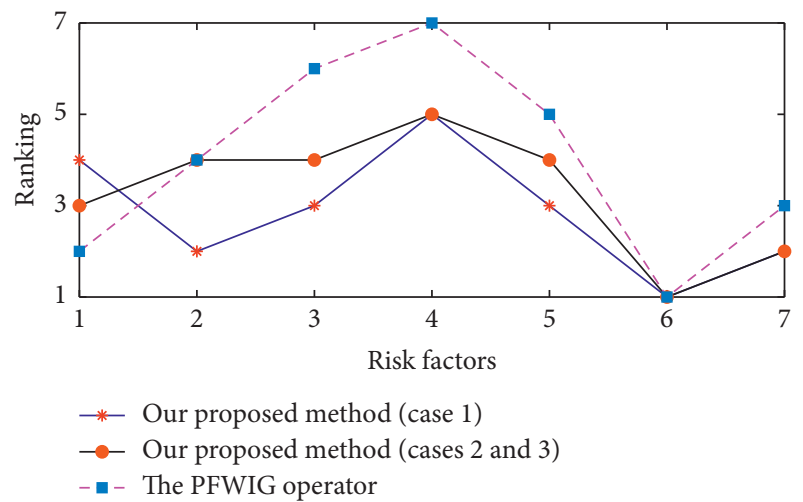

FIGURE 2: The comparison among the results of our proposed method (three cases) and the PFWIG operator method.

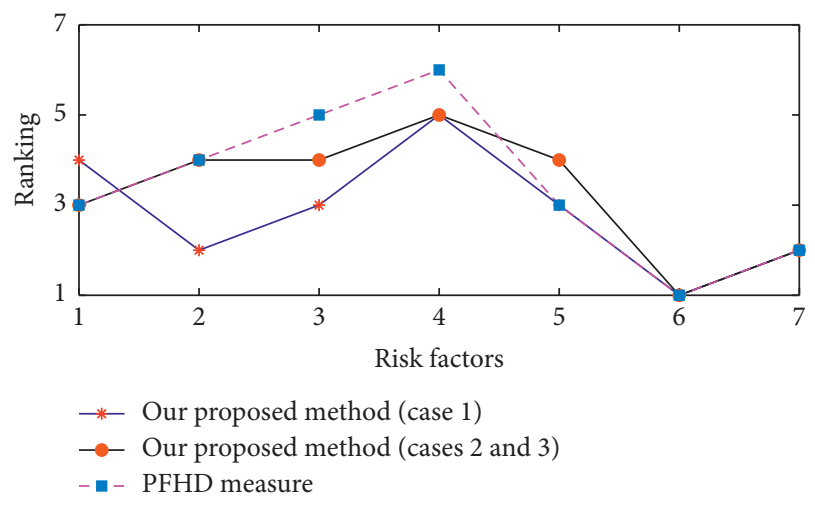

FIGURE 3: The comparison among the results of our proposed method (three cases) and PFHD measure method. 
(1) By using aggregation operators in $[47-49,53,56]$, the ranking results for seven risk factors are $a_{1}=a_{2}=a_{3}=a_{4}=a_{5}=a_{6}=a_{7}$. In addition, the aggregation operators in [55] did not produce any results. Using these methods, we cannot choose the highest risk factor, but through our proposed method, we can choose the highest risk factor $a_{6}$. Therefore, there is a conclusion that our proposed method is more effective and has an extensive application than the selected 15 aggregation operators.

(2) Although the ranking results of our proposed method and these methods in $[3,23,54]$ are different, the highest risk factor and the lowest risk factor are still $a_{6}$ and $a_{4}$, respectively. Therefore, the ranking results derived from our proposed method are valid and credible.

\section{Conclusion and Discussion}

In practical applications, many problems can be described as MCDM problems in a finite PFCAS. In this paper, by combining PF theory, RS theory, and covering theory, a type of CPFRS model is constructed and its properties are discussed. Based on the CPFRS model, we construct a PF TOPSIS method, which can effectively deal with MCDM problems in a finite PFCAS. The advantages of our proposed method are summarized as follows:

(1) The fusion of multiple theories is a highlight of our proposed method. Our proposed method not only enriches the RS theory but also expands the application range of RS theory.

(2) Our proposed method is more effective and has an extensive application than the methods in $[47-49,53,55,56]$. In real life, many decisionmaking problems can be described as the MCDM problems in a finite PFCAS, such as the risk management of green buildings described in Section 4.1. Our proposed method can effectively solve the MCDM problems in a finite PFCAS that cannot be solved by these methods in $[47-49,53,55,56]$.

(3) Although the ranking results of the risk factors using different logical operators described in Section 4.4 are different, the highest risk factor and the lowest risk factor are still $a_{6}$ and $a_{4}$, respectively. This phenomenon shows that our proposed method is feasible and flexible. The decision makers can choose different logical operators according to personal preferences and actual needs when using our proposed method to deal with complex problems.

(4) Since PFS is a direct extension of FS and IFS, our proposed method can also be applied to the MCDM problem in fuzzy or IF environments.

However, our proposed method did not consider the introduction of variable parameters, which makes the proposed method lack certain fault tolerance.
In the future, we plan to further discuss the following topics. (1) We will consider introducing variable parameters into the CPFRS model and investigate covering-based picture fuzzy variable precision rough set model. (2) Due to the importance of the picture fuzzy nano topological spaces [38] in MCDM, we will investigate the topological properties of the CPFRS model. (3) Based on the preference behavior of decision makers, we may combine the CPFRS model with other classical decision-making methods to solve complex economic management problems. (4) We may extend the CPFRS model to different fields to further improve various mathematical models, such as pattern fuzzy recognition and fuzzy cluster analysis.

\section{Data Availability}

No data were used to support this study.

\section{Conflicts of Interest}

The authors declare that there are no conflicts of interest regarding the publication of this paper.

\section{Acknowledgments}

This study was supported by the National Natural Science Foundation of China (no. 11461014) and the Doctoral Starting Up Foundation of Guizhou Normal University (no. GZNUD[2019]13).

\section{References}

[1] L. Huang, C. Wu, B. Wang, and Q. Ouyang, "Big-data-driven safety decision-making: a conceptual framework and its influencing factors," Safety Science, vol. 109, pp. 46-56, 2018.

[2] J. S. Cai, Z. G. Zhang, P. Shi, and H. E. Quan, "Method for risk ranking based on intuitionistic fuzzy multi-attribute group decision-making," Chinese Journal of Engineering Mathematics, vol. 32, no. 5, pp. 650-658, 2015.

[3] L. Wang, H.-y. Zhang, J.-q. Wang, and L. Li, "Picture fuzzy normalized projection-based VIKOR method for the risk evaluation of construction project," Applied Soft Computing, vol. 64, pp. 216-226, 2018.

[4] L. A. Zadeh, "Fuzzy sets," Information and Control, vol. 8, no. 3, pp. 338-353, 1965.

[5] K. T. Atanassov, "Intuitionistic fuzzy sets," Fuzzy Sets and Systems, vol. 20, no. 1, pp. 87-96, 1986.

[6] C.-T. Chen, "Extensions of the TOPSIS for group decisionmaking under fuzzy environment," Fuzzy Sets and Systems, vol. 114 , no. 1 , pp. 1-9, 2000.

[7] Z. $\mathrm{Xu}$ and N. Zhao, "Information fusion for intuitionistic fuzzy decision making: an overview," Information Fusion, vol. 28, pp. 10-23, 2016.

[8] H. Garg and D. Rani, "Novel similarity measure based on the transformed right-angled triangles between intuitionistic fuzzy sets and its applications," Cognitive Computation, 2021.

[9] H. Garg, "Exponential operational laws and new aggregation operators for intuitionistic multiplicative set in multiple-attribute group decision making process," Information Sciences, vol. 538, pp. 245-272, 2020. 
[10] R. R. Yager, "Pythagorean membership grades in multicriteria decision making," IEEE Transactions on Fuzzy Systems, vol. 22, no. 4, pp. 958-965, 2014.

[11] B. Batool, M. Ahmad, S. Abdullah, S. Ashraf, and R. Chinram, "Entropy based pythagorean probabilistic hesitant fuzzy decision making technique and its application for Fog-Haze factor assessment problem," Entropy, vol. 22, no. 3, p. 318, 2020.

[12] A. Khan, S. Ashraf, S. Abdullah, M. Qiyas, J. Luo, and S. Khan, "Pythagorean fuzzy Dombi aggregation operators and their application in decision support system," Symmetry, vol. 11, no. 3, p. 383, 2019.

[13] M. Akram, W. A. Dudek, and F. Ilyas, "Group decisionmaking based on pythagorean fuzzy TOPSIS method," International Journal of Intelligent Systems, vol. 34, no. 7, pp. 1455-1475, 2019.

[14] H. Garg, "Linguistic interval-valued Pythagorean fuzzy sets and their application to multiple attribute group decisionmaking process," Cognitive Computation, vol. 12, no. 6, pp. 1313-1337, 2020.

[15] H. Garg, "Neutrality operations-based Pythagorean fuzzy aggregation operators and its applications to multiple attribute group decision-making process," Journal of Ambient Intelligence and Humanized Computing, vol. 11, no. 7, pp. 3021-3041, 2020.

[16] M. Akram, F. Ilyas, and H. Garg, "Multi-criteria group decision making based on ELECTRE I method in Pythagorean fuzzy information," Soft Computing, vol. 24, no. 5, pp. 3425-3453, 2020.

[17] S. A. Muneeza, S. Abdullah, and M. Aslam, "New multicriteria group decision support systems for small hydropower plant locations selection based on intuitionistic cubic fuzzy aggregation information," International Journal of Intelligent Systems, vol. 35, no. 6, pp. 983-1020, 2020.

[18] F. Smarandache, Neutrosophy: Neutrosophic Probability, Set, and Logic, American Reserch Press, Santa Fe, NM, USA, 1998.

[19] S. Ashraf, S. Abdullah, F. Smarandache, and N. Amin, "Logarithmic hybrid aggregation operators based on single valued neutrosophic sets and their applications in decision support systems," Symmetry, vol. 11, no. 3, p. 364, 2019.

[20] S. Ashraf and S. Abdullah, "Decision support modeling for agriculture land selection based on sine trigonometric single valued neutrosophic information," International Journal of Neutrosophic Science, vol. 9, no. 2, pp. 60-73, 2020.

[21] S. Ashraf, S. Abdullah, S. Zeng, H. Jin, and F. Ghani, "Fuzzy decision support modeling for hydrogen power plant selection based on single valued neutrosophic sine trigonometric aggregation operators," Symmetry, vol. 12, no. 2, p. 298, 2020.

[22] C. Zhang, D. Y. Li, X. P. Kang, D. Song, A. K. Sangaish, and S. Broumi, "Neutrosophic fusion of rough set theory: an overview," Computers in Industry, vol. 115, Article ID 103117, 2020.

[23] B. C. Cuong, "Picture fuzzy sets," Journal of Computer Science and Cybernetics, vol. 30, no. 4, pp. 409-420, 2014.

[24] S. Ashraf and S. Abdullah, "Spherical aggregation operators and their application in multiattribute group decision-making," International Journal of Intelligent Systems, vol. 34, no. 3, pp. 493-523, 2019.

[25] S. Ashraf, S. Abdullah, and T. Mahmood, "GRA method based on spherical linguistic fuzzy Choquet integral environment and its application in multi-attribute decision-making problems," Mathematical Sciences, vol. 12, no. 4, pp. 263-275, 2018.
[26] S. Ashraf, S. Abdullah, and M. Aslam, "Symmetric sum based aggregation operators for spherical fuzzy information: application in multi-attribute group decision making problem," Journal of Intelligent \& Fuzzy Systems, vol. 38, no. 4, pp. 5241-5255, 2020.

[27] S. Ashraf, S. Abdullah, and T. Mahmood, "Spherical fuzzy Dombi aggregation operators and their application in group decision making problems," Journal of Ambient Intelligence and Humanized Computing, vol. 11, no. 7, pp. 2731-2749, 2020.

[28] S. Ashraf, S. Abdullah, T. Mahmood, F. Ghani, and T. Mahmood, "Spherical fuzzy sets and their applications in multi-attribute decision making problems," Journal of Intelligent \& Fuzzy Systems, vol. 36, no. 3, pp. 2829-2844, 2019.

[29] Y. Jin, S. Ashraf, and S. Abdullah, "Spherical fuzzy logarithmic aggregation operators based on entropy and their application in decision support systems," Entropy, vol. 21, no. 7, p. 628, 2019.

[30] S. Ashraf, S. Abdullah, M. Aslam, M. Qiyas, and M. A. Kutbi, "Spherical fuzzy sets and its representation of spherical fuzzy t-norms and t-conorms," Journal of Intelligent \& Fuzzy Systems, vol. 36, no. 6, pp. 6089-6102, 2019.

[31] S. Ashraf, S. Abdullah, and L. Abdullah, "Child development influence environmental factors determined using spherical fuzzy distance measures," Mathematics, vol. 7, no. 8, p. 661, 2019.

[32] H. Jin, S. Ashraf, S. Abdullah, M. Qiyas, M. Bano, and S. Zeng, "Linguistic spherical fuzzy aggregation operators and their applications in multi-attribute decision making Problems," Mathematics, vol. 7, no. 5, p. 413, 2019.

[33] S. Ashraf and S. Abdullah, "Emergency decision support modeling for COVID-19 based on spherical fuzzy information," International Journal of Intelligent Systems, vol. 35, no. 11, pp. 1601-1645, 2020.

[34] M. Rafiq, S. Ashraf, S. Abdullah, T. Mahmood, and S. Muhammad, "The cosine similarity measures of spherical fuzzy sets and their applications in decision making," Journal of Intelligent \& Fuzzy Systems, vol. 36, no. 6, pp. 6059-6073, 2019.

[35] O. Barukab, S. Abdullah, S. Ashraf, M. Arif, and S. A. Khan, "A new approach to fuzzy TOPSIS method based on entropy measure under spherical fuzzy information," Entropy, vol. 21, no. 12, p. 1231, 2019.

[36] X. T. Nguyena and S. Florentin, “( $\mathscr{I}, \mathscr{T})$-Standard neutrosophic rough set and its topologies properties," Neutrosophic Sets and Systems, vol. 14, pp. 65-70, 2016.

[37] L. H. Son, "Measuring analogousness in picture fuzzy sets: from picture distance measures to picture association measures," Fuzzy Optimization and Decision Making, vol. 16, pp. 1-20, 2017.

[38] I. Alshammari, P. Mani, C. Ozel, and H. Garg, "Multiple attribute decision making algorithm via picture fuzzy nano topological spaces," Symmetry, vol. 13, no. 1, p. 69, 2021.

[39] G. Wei, "Picture fuzzy cross-entropy for multiple attribute decision making problems," Journal of Business Economics and Management, vol. 17, no. 4, pp. 491-502, 2016.

[40] D. Joshi and S. Kumar, "An approach to multi-criteria decision making problems using dice similarity measure for picture fuzzy sets," in Proceedings of the International Conference on Mathematics and Computing, pp. 135-140, Varanasi, India, January 2018.

[41] X. Peng and J. Dai, "Algorithm for picture fuzzy multiple attribute decision-making based on new distance measure," 
International Journal for Uncertainty Quantification, vol. 7, no. 2, pp. 177-187, 2017.

[42] L. Wang, J.-j. Peng, and J.-q. Wang, "A multi-criteria decision-making framework for risk ranking of energy performance contracting project under picture fuzzy environment," Journal of Cleaner Production, vol. 191, pp. 105-118, 2018.

[43] G. Wei, "TODIM method for picture fuzzy multiple attribute decision making," Informatica, vol. 29, no. 3, pp. 555-566, 2018.

[44] S. Ashraf, T. Mahmood, S. Abdullah, and Q. Khan, "Different approaches to multi-criteria group decision making problems for picture fuzzy environment," Bulletin of the Brazilian Mathematical Society, New Series, vol. 50, no. 2, pp. 373-397, 2019.

[45] Z. L. Yang, X. Li, H. Garg, R. Peng, S. M. Wu, and L. C. Huang, "Group decision algorithm for aged healthcare product purchase under q-rung picture normal fuzzy environment using Heronian mean operator," International Journal of Computational Intelligence Systems, vol. 13, no. 1, pp. 11761197, 2020.

[46] M. Akram, A. Bashir, and H. Garg, "Decision making model under complex picture fuzzy hamacher aggregation operators," Computational and Applied Mathematics, vol. 39, p. 226, 2020.

[47] H. Garg, "Some picture fuzzy aggregation operators and their applications to multicriteria decision-making," Arabian Journal for Science and Engineering, vol. 42, no. 12, pp. 5275-5290, 2017.

[48] G. Wei, "Picture fuzzy aggregation operators and their application to multiple attribute decision making," Journal of Intelligent \& Fuzzy Systems, vol. 33, no. 2, pp. 713-724, 2017.

[49] C. Y. Wang, "Hesitant fuzzy set and picture fuzzy set with their application research," Ph.D. thesis, Hunan University, Hubei, China, 2015.

[50] C. Y. Wang, X. Q. Zhou, H. N. Tu, and S. D. Tao, "Some geometric aggregation operators based on picture fuzzy sets and their application in multiple attribute decision making," Italian Journal of Pure and Applied Mathematics, vol. 37, pp. 477-492, 2017.

[51] R. Wang, J. Wang, H. Gao, and G. Wei, "Methods for MADM with picture fuzzy Muirhead mean operators and their application for evaluating the financial investment risk," Symmetry, vol. 11, no. 1, p. 6, 2018.

[52] C. Tian, J. J. Peng, S. Zhang, W. Y. Zhang, and J. Q. Wang, "Weighted picture fuzzy aggregation operators and their applications to multi-criteria decision-making problems," Computers \& Industrial Engineering, vol. 137, Article ID 106037, 2019.

[53] X. Li, Y. Ju, D. Ju, W. Zhang, P. Dong, and A. Wang, "Multiattribute group decision making method based on edas under picture fuzzy environment," IEEE Access, vol. 7, pp. 141179141192, 2019.

[54] Y. Ju, D. Ju, E. D. R. Santibanez Gonzalez, M. Giannakis, and A. Wang, "Study of site selection of electric vehicle charging station based on extended GRP method under picture fuzzy environment," Computers \& Industrial Engineering, vol. 135, pp. 1271-1285, 2019.

[55] C. Jana, T. Senapati, M. Pal, and R. R. Yager, "Picture fuzzy Dombi aggregation operators: application to MADM process," Applied Soft Computing, vol. 74, pp. 99-109, 2019.

[56] G. W. Wei, "Picture fuzzy Hamacher aggregation operators and their application to multiple attribute decision making," Fundamenta Informaticae, vol. 157, no. 3, pp. 271-320, 2018.

[57] Z. a. Pawlak, "Rough sets," International Journal of Computer \& Information Sciences, vol. 11, no. 5, pp. 341-356, 1982.
[58] W. Zakowski, "Approximations in the space $(U, \pi)$," Demonstratio Mathematica, vol. 16, no. 3, pp. 761-769, 1983.

[59] W.-H. Xu and W.-X. Zhang, "Measuring roughness of generalized rough sets induced by a covering," Fuzzy Sets and Systems, vol. 158, no. 22, pp. 2443-2455, 2007.

[60] D. Dubois and H. Prade, "*Rough fuzzy sets and fuzzy rough sets," International Journal of General Systems, vol. 17, no. 2-3, pp. 191-209, 1990.

[61] L. Ma, "Two fuzzy covering rough set models and their generalizations over fuzzy lattices," Fuzzy Sets and Systems, vol. 294, pp. 1-17, 2016.

[62] L. D'eer and C. Cornelis, "A comprehensive study of fuzzy covering-based rough set models: defintions, properties and interrelationships," Fuzzy Sets and Systems, vol. 336, pp. 1-26, 2018.

[63] J. Zhan, B. Sun, and J. C. R. Alcantud, "Covering based multigranulation (I, T)-fuzzy rough set models and applications in multi-attribute group decision-making," Information Sciences, vol. 476, pp. 290-318, 2019.

[64] J. Ye, J. Zhan, W. Ding, and H. Fujita, "A novel fuzzy rough set model with fuzzy neighborhood operators," Information Sciences, vol. 544, pp. 266-297, 2021.

[65] L. Zhang, J. Zhan, and Y. Yao, "Intuitionistic fuzzy TOPSIS method based on CVPIFRS models: an application to biomedical problems," Information Sciences, vol. 517, pp. 315-339, 2020.

[66] L. Zhang, J. Zhan, and Z. Xu, "Covering-based generalized IF rough sets with applications to multi-attribute decisionmaking," Information Sciences, vol. 478, pp. 275-302, 2019.

[67] S. Zeng, A. Hussain, T. Mahmood, M. Irfan Ali, S. Ashraf, and M. Munir, "Covering-based spherical fuzzy rough set model hybrid with TOPSIS for multi-attribute decision-making," Symmetry, vol. 11, no. 4, p. 547, 2019.

[68] M. Akram, Shumaiza, and M. Arshad, "Bipolar fuzzy TOPSIS and bipolar fuzzy ELECTREI methods to diagnosis," Computational and Applied Mathematics, 2020.

[69] M. Akram, S. Naz, and F. Smarandache, "Generalization of maximizing deviation and TOPSIS method for MADM in simplified neutrosophic hesitant fuzzy environment," Symmetry, vol. 11, no. 8, p. 1058, 2019.

[70] B. C. Cuong, V. Kreinovitch, and R. T. Ngin, "A classification of representablet-norm operators for picture fuzzy sets," in Proceedings of the 8th International Conference on Knowledge and Systems Engineering (KSE), pp. 19-24, Hanoi, Vietnam, Octopber 2016.

[71] B. C. Cuong and P. V. Hai, "Some fuzzy logic operators for picture fuzzy sets," in Proceedings of the 7th International Conference on Knowledge and Systems Engineering (KSE), pp. 132-137, Ho Chi Minh City, Vietnam, Octopber 2015.

[72] B. C. Cuong, L. V. Hung, and P. V. Chien, "Picture fuzzy rough sets: some new basic propositions," in Proceedings of the 4th NAFOSTED Conference on Information and Computer Science, pp. 48-53, Hanoi, Vietnam, November 2017.

[73] L. D'eer, C. Cornelis, and L. Godo, "Fuzzy neighborhhod operators based on fuzzy covering," Fuzzy Sets and Systems, vol. 312, pp. 17-35, 2017.

[74] Y. Yao and B. Yao, "Covering based rough set approximations," Information Sciences, vol. 200, no. 1, pp. 91-107, 2012.

[75] A. M. Radzikowska and E. E. Kerre, "A comparative study of fuzzy rough sets," Fuzzy Sets and Systems, vol. 126, no. 2, pp. 137-155, 2002.

[76] L. Zhou, W.-Z. Wu, and W.-X. Zhang, "On characterization of intuitionistic fuzzy rough sets based on intuitionistic fuzzy implicators," Information Sciences, vol. 179, no. 7, pp. 883-898, 2009. 